\title{
Que peut apporter une modélisation mathématique à la maitrise du bio-printing?
}

\section{What can mathematical modelling contribute to the mastery of bio-} printing?

\author{
Danzo A. ${ }^{1}$, André J.C. ${ }^{1}$ \\ ${ }^{1}$ LRGP - UMR 7274 CNRS-UL, 1, rue Grandville - F54000 Nancy, Jean-claude.andre@univ-lorraine.fr
}

RÉSUMÉ. Le bio-printing issu des technologies de fabrication additive se développe en termes de recherches scientifiques parce qu'avec cette technologie récente il y aurait possibilité, avec des cellules souches des personnes atteintes de maladies graves, de recréer un jour des organes déficients sans qu'existent des rejets de la part des patients malades puisque ces organes seraient recréés à partir de cellules saines compatibles. Ce que l'on observe c'est une augmentation quasi-exponentielle du volume des publications expérimentales alors que la modélisation des processus de croissance et de différenciation cellulaire reste peu explorée. Pour autant, le bio-printing nécessite la convergence de nombreuses disciplines scientifiques, ce qui impose une réflexion épistémologique visant la fabrication robuste d'organes. Faute de la possibilité de rencontre entre spécialistes scientifiques concernés, la modélisation mathématique permet de tenter de trouver de possibles chemins existant entre extraction de cellules saines d'un patient et réalisation potentielle d'un tissu, voire d'un organe. Le but de ce travail est d'illustrer cette potentialité permettant de savoir quelles questions poser aux biologistes pour avancer sur ce sujet (en particulier), mais également de connaître les limites de ce type d'exercice.

ABSTRACT. Bio-printing from additive manufacturing technologies is developing in terms of scientific research because with this recent technology it would be possible, with stem cells from people with serious diseases, to one day recreate deficient organs without rejection from sick patients since these organs would be recreated from compatible healthy cells. What is observed is an almost exponential increase in the volume of experimental publications, while the modelling of cell growth and differentiation processes remains little explored. Nevertheless, bioprinting requires the convergence of many scientific disciplines, which imposes an epistemological reflection aimed at robust organ production. In the absence of the possibility of a deep association between the scientific specialists concerned, mathematical modelling makes it possible to attempt to find possible paths existing between the extraction of healthy cells from a patient and the potential production of a tissue, or even an organ. The aim of this work is to illustrate this potentiality, making it possible to know what questions to ask biologists in order to make progress on this subject (in particular), but also to know the limits of this type of exercise.

MOTS-CLÉS. Fabrication additive, bio-printing, épistémologie, modélisation mathématique.

KEYWORDS. Additive manufacturing, bio-printing, epistemology, mathematical modelling.

Le bio-printing est une technique de fabrication additive permettant d'imprimer en trois dimensions des couches successives de cellules sur des bio-matrices afin de reconstituer à l'identique la structure d'un organe entier (cf. par exemple André, 2017 ; Guédon, Malaquin et André, 2017 ; 2017 a ; Peels, 2019). Le bio-printing a déjà été utilisé pour produire des éléments de la peau, des os, des greffons vasculaires, des attelles trachéales, du tissu cardiaque et des structures cartilagineuses, etc. Bien qu'encore très récente, cette méthode est très prometteuse, non seulement pour le domaine de la médecine régénérative, mais aussi pour la découverte de médicaments et la recherche en toxicologie.

Pour créer une vie d'origine partiellement artificielle simulant ce que sait faire la Nature et même aller au-delà, il faudrait en principe disposer de connaissances approfondies sur la vie naturelle. Cependant, il est possible d'imaginer qu'à partir de concepts " rudimentaires " on s'élèvera progressivement d'essais de laboratoire à des réparations de tissus ou d'organes biologiques (Parrochia, 1994). Le bio-printing appartient à l'ingénierie du vivant ou à la bio-ingénierie, qui intègre les sciences physiques, chimiques, mathématiques, ainsi que les principes d'ingénierie pour 
étudier la biologie, la médecine, les comportements et la santé : il vise la fabrication d'organes vivants. Il constitue une forme de chaînon manquant entre l'artificiel et le naturel. Cette définition appelle une remarque : le bio-printing ne peut pas être adapté à l'ensemble des systèmes vivants, mais à des situations personnalisées et spécifiques (d'un tissu, par exemple) exploitant des systèmes artificiels...

La figure 1 représente l'évolution (exponentielle) des nombres de publications au cours du temps sur ce sujet disruptif à cause de la possibilité d'envisager la fabrication de tissus mous dont le besoin applicatif en médecine est presqu'évident pour tout un chacun.

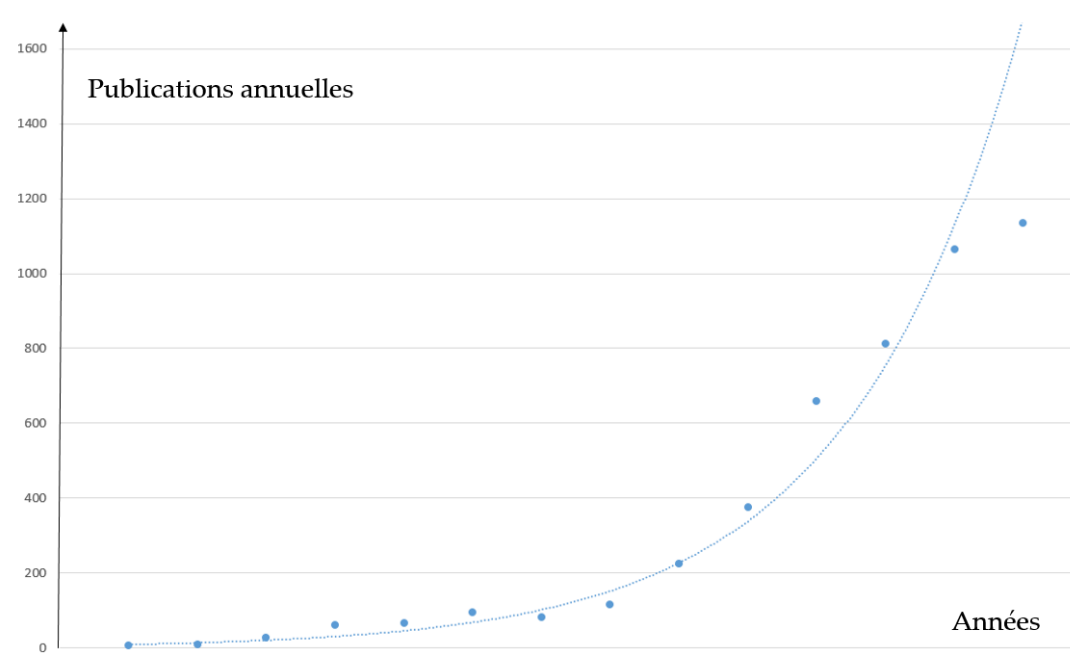

20062007200820092010201120122013201420152016201720182019

Figure 1. Evolution du nombre de publications dans le domaine du bio-printing (source Bibliothèque du CNRS)

Avec l'individualisme, la spécificité des corps risque de devenir l'un des fondements de la médecine de précision de demain, en tant qu'elle permet des soins optimisés (et moins coûteux en principe). Dans le même temps, les effets de la transformation du concept de santé (OMS) allant de la suppression de la maladie, à la réalisation d'un bien-être individuel (médecine de confort) soutiennent le développement potentiel d'« human enhancement », développement autorisé dans son principe par le bio-printing. De plus, l'avènement de l'impression 3D pourrait résoudre certains des problèmes les plus pressants auxquels fait face le don d'organes - comme la disponibilité et le risque de rejet par le corps du patient. Au lieu d'utiliser de l'encre, ces «imprimantes » d'organes déposent donc un mélange de cellules vivantes (« encre-bio » ou «bio-ink»), couche par couche, pour former des tissus humains. Comme les organes sont imprimés avec les cellules du patient, elles ne sont en principe pas rejetées par le corps du patient. Le succès de l'impression d'organes humains utilisables semble donc prometteur et fortement désirable...

Des échanges entre des spécialités de biologie (biochimie, biologie moléculaire, biologie cellulaire, bio-informatique) et les spécialités qui fabriquent, observent ou caractérisent des objets issus des technologies de fabrication additive (selon les projets, chimie, physique appliquée, mécanique, électronique, bio-informatique, science des matériaux, traitement du signal, automatique, photonique, génie des procédés, etc.) visent à enrichir les connaissances et les capacités d'action sur la «fabrication du vivant ». Ces scientifiques parlent tranquillement d'un monde nouveau, fascinant et un peu inquiétant ; celui de l'ingénierie des cellules souches, des biomatériaux, du micro-patterning et du bio-printing, des bioréacteurs et de modélisation du vivant. Ils affirment que nous sommes déjà en partie capables de reconstruire nos organes, foie (Samsara, 2016), reins, peau, cœur et pourquoi pas cerveau à partir de nos cellules souches et en impression $3 \mathrm{D}$ et nous expliquent comment les scientifiques, partout dans le monde, avancent à pas de géants sur ce terrain du bio-engineering, du bio-printing et de la reconstruction du vivant... Mais de quel 
vivant parle-t-on ? S'appuie-t-on sur les seuls critères classiques suivants : autoreproduction, fonction, communication et évolution ? Et avec quels degrés ? Doit-on, ou non, se méfier des premiers résultats et des analogies séduisantes ?

Sur ces bases, la vision initiale de l'ingénieur a été de faire simple, si possible à l'intérieur d'une pensée causaliste, avec les outils à sa disposition. La matière vivante peut-être ordonnée, signifiant que sa forme peut-être, au moins en première approximation, décrite géométriquement. On peut penser que les structures, plus ou moins auto-organisées, doivent être associées à certains types de conditions physiques ou physico-chimiques. En se plaçant dans une perspective évolutionnaire plus large, il est toutefois impossible de prédire l'évolution des formes en les déduisant de l'application des lois simples que nous aurions pu identifier. Plus exactement, à l'expérience, si ces lois sont nécessaires, elles ne sont pas suffisantes. Elles forment l'arrière-plan incontournable d'une évolution qui se développe de façon non apparemment déterministe par interaction des systèmes naturels entre eux et avec leur environnement. Les sciences de la «morphogenèse » n'auraient donc de validité que dans les limites de la physique ordinaire ou macroscopique. Or, celle-ci n'est qu'une approximation imposant de chasser le déterminisme linéaire...

En préparant des éléments de formes par fabrication additive (incluant matière inerte et matière vivante) pour imposer une structure et une forme (réduction de la maille de travail), il est possible de penser qu'on pourra arriver (demain) à des réparations d'organes en introduisant là où il faut et quand il faut des cellules vivantes qui sauront se développer pour une fin donnée (avec un environnement connu). Dans ce contexte "paradigmatique », le bio-printing se situerait entre la fabrication additive «classique », domaine d'excellence des ingénieurs (procédés et matériaux) et la biologie, voire les sciences de la vie « naturelle » (si l'on n'est pas à une tautologie près). Guidolin, Rebuffat et Albertin (2011) proposent un flow-chart classique partiellement adapté à la situation du bio-printing présenté sur la figure 2 . Cet aspect modélisation est de fait LA difficulté du domaine.

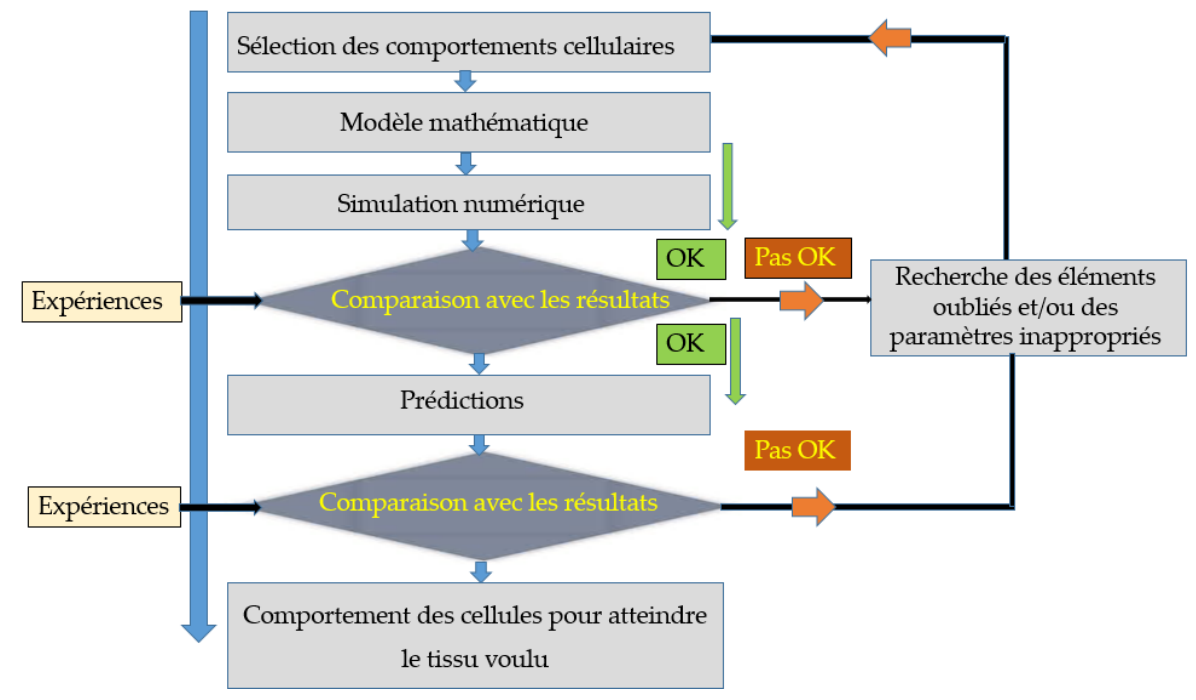

Figure 2. Flow-chart traditionnel adapté au bio-printing

Recherche des éléments oubliés et/ou des paramètres inappropriés OK/Pas OK

Cependant, la réduction présentée dans cette figure qui est un peu d'obédience déterministe avec des relations causes-effets identifiées, est probablement suffisante pour qu'on reste à un niveau de généralité de cadrage de phénomènes évolutifs. C'est que la non connaissance des rôles d'éléments biologiques, des effets récursifs et associatifs entre systèmes cellulaires a suffisamment d'influence pour rendre caduc cette base classique d'approche scientifique (le modèle proposé ne fera probablement pas avancer la compréhension des systèmes complexes spatio-temporels à étudier)... Néanmoins, sur la seule année 2019 de nombreux progrès ont été mentionnés dans les domaines 
extrêmement larges offert par le concept de bio-printing (cf. par exemple : Lin et al, 2019 ; Atala et Forgacs, 2019; Wimmer et al, 2019; Jiang et al, 2019; Bittner et al, 2019; Osaghae, 2019; Grigoryan et al, 2019 ; Landau et al, 2019 ; Esmaeili et al, 2019 ; Chen et al, 2019 ; Hanumantharao, Que et Rao, 2019 ; Zengh et al, 2020 ; Tamay et al, 2019 ; Li et al, 2019 ; Seth et al, 2017 ; SkylarScott et al, 2019 ; Senior et al, 2019 ; Bruno, Reid et Sachs, 2019 ; Listek, 2019 ; Baltazar et al, 2019 ; Karabay et al, 2019 ; Godesky et Shreibera, 2019 ; etc.).

\section{Technologies du bio-printing : de la cellule à l'organe}

Le bio-printing pour Peng, Unutmaz et Ozbolat (2016) dispose de nombeux avantages de principe relativement aux autres méthodes de bio-fabrication comme le moulage, les assemblages par voies magnétiques et les approches micro-fluidiques pour les raisons suivantes :

- Il permet de réaliser des tissus bio-imprimés conformes à ce qui est attendu des points de vue géométrie et (partiellement) fonctionnalité ;

- Le bio-printing permet la fabrication de structures poreuses (sur lesquelles les cellules peuvent s'accrocher) avec une architecture maîtrisée ;

- Il autorise des co-cultures avec de multiples types de cellules;

- Il permet d'associer sur des systèmes modèles des cellules et des ajouts divers ;

- Le bio-printing permet la délivrance de facteurs de croissance et de gènes;

- Il est flexible et autorise la fabrication de tissus « à façon »;

- L'intégration de la vascularisation est possible (Richards et al, 2017) ;

- Les bio-encres utilisées sont de quatre sortes : des hydrogels contenant des cellules, des micro-transporteurs, des aggrégats cellulaires, des matrices dé-cellularisées.

- En remarque : rien n'est siganlé par ces auteurs sur l'histoire cellulaire induite (vieillissement différentiel) par le procédé de fabrication lui-même : pour une durée longue (plusieurs heures) de fabrication, les cellules imprimées en fin d'opération auront un environnement différent de celui des cellules déposées en tout début, ce qui peut avoir une incidence sur le comportement futur des cellules.

La figure 3 présente le principe général retenu pour une réparation sur 1'Homme à partir de ses propres cellules.

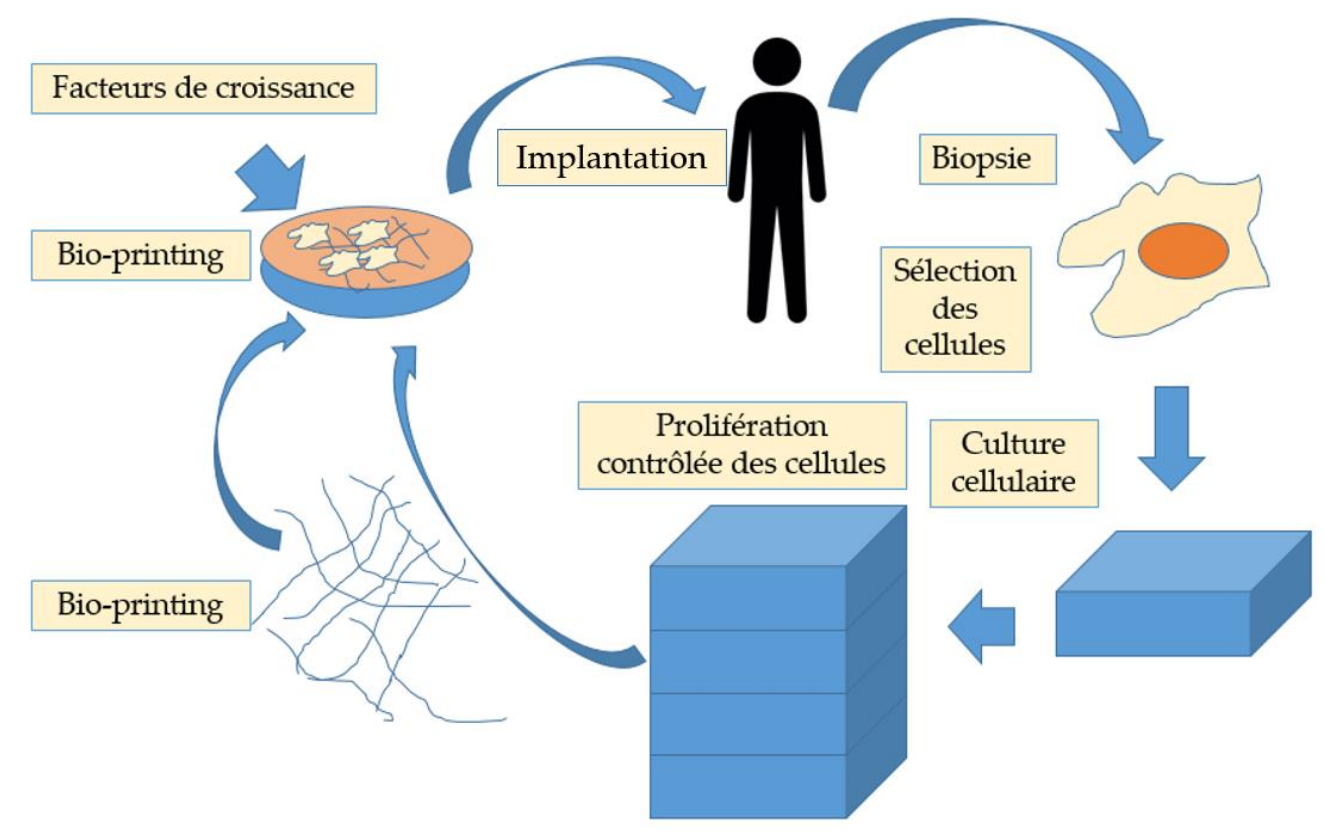

Figure 3. Principe général d'utilisation (" cercle vertueux ») du bio-printing 
Selon Chneiweiss (2012), plusieurs conditions doivent être satisfaites :

- Les cellules doivent être suffisamment différenciées pour remplir spécifiquement la fonction demandée ;

- Elles doivent être capables de survivre pour occuper l'espace fourni par l'impression 3D et de rester en vie pour remplir la fonction de l'organe ;

- Elles doivent rester dans l'état voulu (le mécanisme qui a servi à les orienter est-il stable ? ya-t-il de possibles bifurcations ? des risques d'échappement ?) ;

- Elles doivent être capables d'établir les contacts fonctionnels nécessaires avec leur environnement (rétablissement de la fonction du réseau cellulaire).

Il s'agit, malgré des réserves ou des contraintes à maîtriser, d'une situation émergente particulièrement « excitante », réel défi scientifique et applicatif, qui concerne la réalisation d'objets soit en matière vivante, soit comme support pour des réparations diverses. Derrière le Bio-printing se cache l'idée de pouvoir modéliser le vivant en pensant qu'il sera possible de passer outre l'incomplétude immense de nos connaissances sur ce monde. L'impression d'éléments vivants de toutes natures relève d'une technique classique de fabrication additive appliquée à de la matière vivante, mais ce n'est pas trop la technologie qui est problématique, c'est son utilisation avec des cellules vivantes qui explore la complexité des phénomènes de la Nature...

Une question initiale à ce stade est de savoir si la richesse cellulaire d'un tissu ou d'un organe doit être introduite dans le processus de bio-printing (ce qui parait pour le moins délicat, cf. Sommer, 2016), ou si l'on se « satisfait » d'un lot réduit d'espèces cellulaires qui auront pour « mission »de proliférer et de se différencier ultérieurement pour atteindre l'objectif prévu par le chercheur. Cette première remarque constitue à l'évidence un des verrous à faire sauter pour avancer dans ce domaine.

Dans les faits, une simplification est opérée par l'utilisation de cellules souches indifférenciées. L'idée est donc simple dans son principe: implanter des cellules souches qui pourraient se transformer pour produire un bio-construit satisfaisant grâce à la bonne différenciation cellulaire. Toutefois, il existe un certain nombre de limitations: technologiques (évoquées ci-après) ; scientifiques pour savoir comment orienter la transformation en vue d'une telle fin, hétérogénéité des tissus (angiogénèse, vaisseaux, nerfs, etc.), changements d'échelle (des compartiments de la cellule à l'humain), compatibilités diverses, réglementation, éthique, la formation du corps médical, etc. Or, d'un point de vue technologique, le dépôt « simple » de cellules ne permet pas leur survie par manque d'un flux de nutriments et d'oxygène suffisant. Une seconde question émerge alors, celle liée à la présence d'échafaudages (scaffolds) sur lesquels les cellules vont être déposées, question qui nécessite pour une réponse acceptable un «bon » choix de matériaux et un design adapté pour que les cellules déposées se développent... Selon les situations, les amplitudes des réponses peuvent être variables.

Dans les travaux de bio-impression actuels (en nombre encore limité, mais en très forte augmentation comme le montre la figure 1) on tente de montrer qu'il est possible de trouver des tendances favorables, malgré la complexité des tissus, laissant à penser (mais c'est à confirmer) que la présence des « bonnes » cellules peut être responsable d'une « bonne » réparation par ce procédé de bio-fabrication. En effet, tout le monde connaît la faculté qu'ont les lézards à faire repousser leur queue, les tritons des membres, etc. grâce à leurs cellules souches totipotentes. La nature offre cependant beaucoup moins de possibilités de ce genre aux vertébrés supérieurs que sont les mammifères (Kahn et Papillon, 2005). La question posée ici est de savoir s'il existe un curseur et de maîtriser son positionnement pour qu'un tissu soit fabricable et corresponde à ce que l'on attend de lui. C'est à ce prix que le bio-printing pourra concurrencer les autres technologies émergentes que sont la médecine régénérative et la biologie de synthèse, ou plus simplement la Nature (cf. par exemple, Park et al 2015 ; Ozbolat, Peng et Ozbolat, 2016 ; Lei et Wang, 2016). 
L'idée générique du bio-printing est représentée sur la figure $4:$ placer sur un support les «bonnes » cellules, sur un "bon » échafaudage pour qu'un tissu vivant puisse se développer (Melchels et al, 2012 ; André, 2017 a ; Sun et al, 2020). Au nom du bio-printing, des chercheurs s'appliquent à construire le plus intelligemment possible des "briques de vivant ", voxels complexes et à la fois très simplifiés et contrôlables, qu'il suffirait ensuite "d'empiler » pour obtenir des tissus viables répondant à un cahier des charges fonctionnel (et pourquoi pas des êtres vivants plus compétitifs !). Sur la figure 5 qui provient dans l'esprit de la même origine, le développement cellulaire s'opérationnalise autour d'un flux de matière et d'énergie.

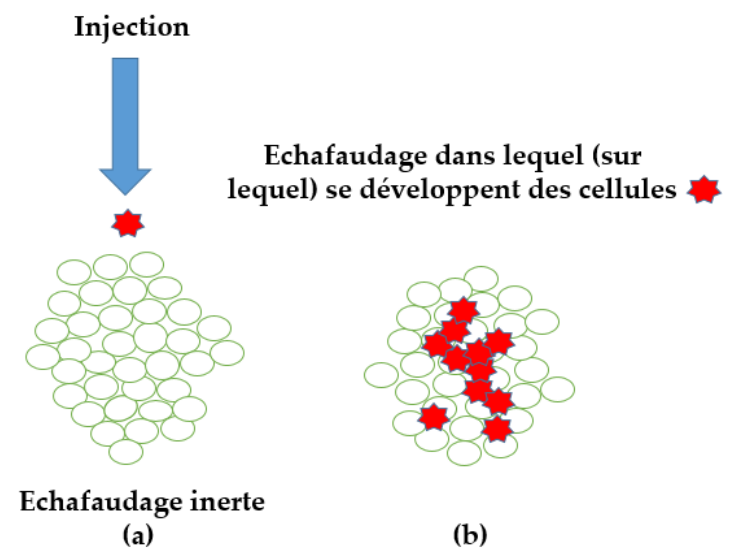

Figure 4. Association support - dépôt de cellules dans le bio-printing

(a)

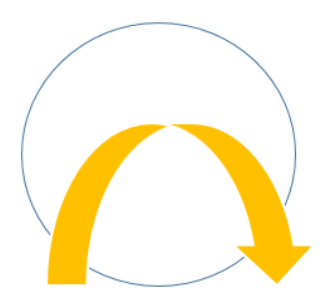

(b)

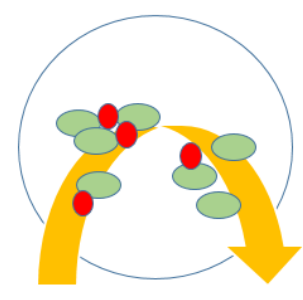

(c)

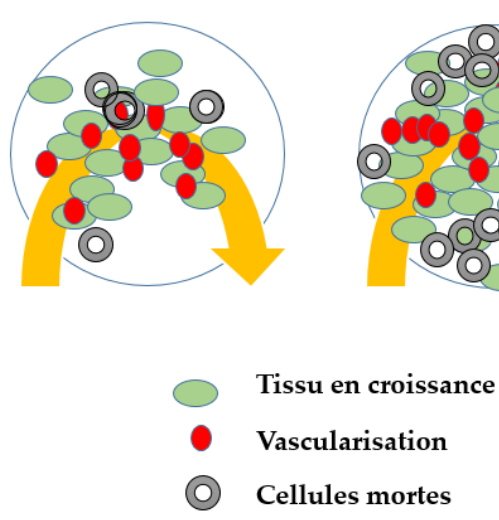

(d)

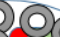

(o)

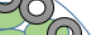

.

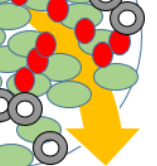

\author{
(1)
}




\section{Du besoin de convergence au modèle de tendance}

Selon Lipson et Kurman (2014), jusqu'à présent, la Nature est restée bien plus performante que l'être humain et l'ordinateur pour ce qui est de choisir et de disposer les différentes cellules souches de manière optimale ! Présentée comme domaine éminemment collaboratif, le bio-printing, de par la nécessité d'intégrer des principes de convergence (MIT, 2016), offre un terrain d'observation particulièrement intéressant de l'interdisciplinarité. Sur cette base des alliances disciplinaires doivent, après apprentissage d'un langage de communication commun, permettre de réfléchir aux meilleures façons d'explorer ce domaine porteur sans qu'on puisse aujourd'hui savoir si l'on saura définir une relation causale entre projet (de réparation, et pourquoi pas d'augmentation humaine) et fonctionnalité attendue. De fait, la consultation de différents partenaires issus de disciplines différentes doit s'exprimer à travers une diversité de courants révélatrice de la dimension, de la complexité et des tensions du domaine émergent qu'est le bio-printing. Il en résulte normalement des difficultés de " coopération » entre des acteurs fortement hétérogènes, mais c'est peut-être surmontable, permettant d'examiner si les difficultés sont ailleurs.

Le terme « technologies convergentes » vise à intégrer dans un système technique des composants ou des concepts développés séparément. La fertilisation croisée des technologies convergentes serait source d'innovations (Laurent, 2005). Pour l'AFTU (2006), il s'agit bien d'une approche interdisciplinaire. L'idée de base de la convergence c'est bien d'extraire de savoirs disjoints des voies permettant par fusion de savoirs disciplinaires des applications industrielles, ce qui induit un couplage entre des aspects scientifiques, économiques et sociaux pour une innovation réussie. Compte-tenu de ce qui vient d'être précisé, le domaine du bio-printing doit exploiter différents supports disciplinaires comme l'indique la figure 6.

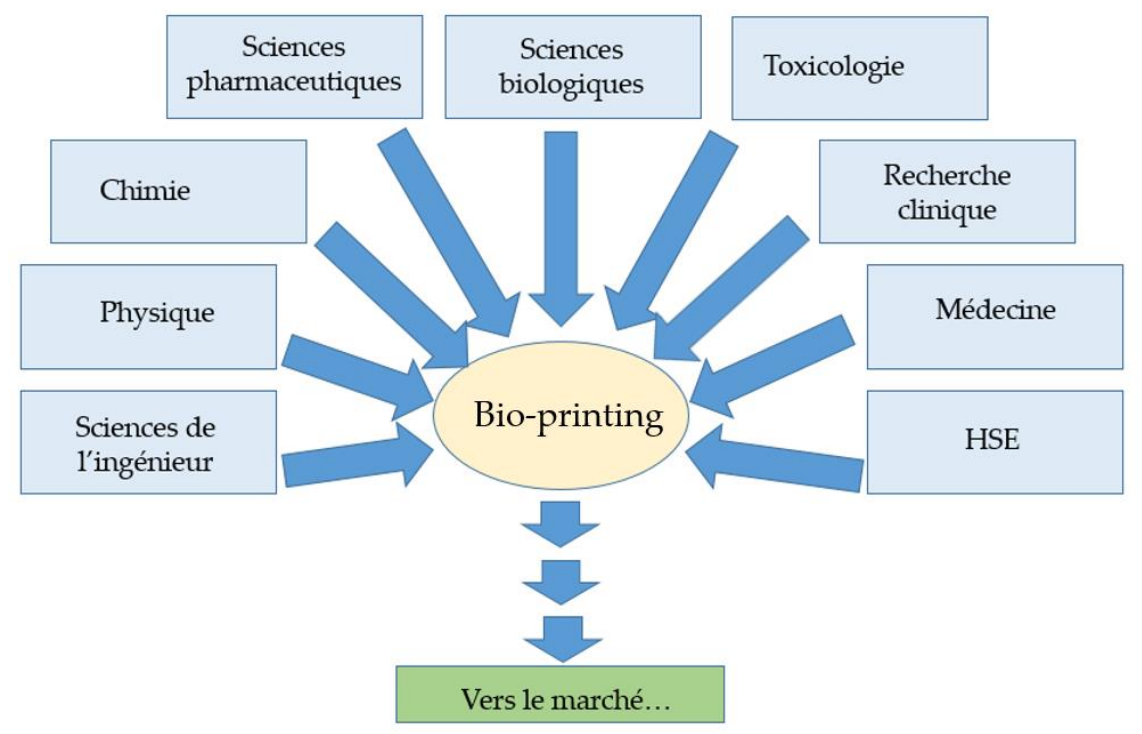

Figure 6. Convergences BP (pour bio-printing) et approche interdisciplinaire (HSE pour Hygiène, Sécurité et Environnement)

Knorr-Cetina (1999) définit des cultures épistémiques comme des machines à produire des faits, ce qui nécessite des scientifiques « habiles »..., des ontologies d'organismes et des « machines » qui résultent de connaissances issues de raisonnements par analogie, issues de différents champs scientifiques et techniques, des maîtrises des verrous à faire sauter, etc. Ainsi, l'épistémologie à créer entre du virtuel (modèles réalistes) et le réel simplifié demande à être construite à partir de différentes disciplines avec des zones de transaction ; cette épistémologie itérative (instationnaire) résulte d'un jeu interdisciplinaire évident. Ainsi, présenté comme domaine éminemment collaboratif, le bio-printing devrait offrir un terrain d'observation particulièrement intéressant de l'interdisciplinarité. Sur cette base des alliances disciplinaires doivent permettre de réfléchir aux 
meilleures façons d'explorer ces domaines considérés comme porteurs d'espoirs, sans qu'on puisse aujourd'hui savoir si l'on saura définir une relation efficiente entre projet BP et fonctionnalité attendue. De fait, la consultation de différents partenaires issus de disciplines différentes devrait s'exprimer à travers une diversité de courants révélatrice de la dimension, de la complexité et des tensions de ce domaine émergent. Il doit en résulter normalement des difficultés de « coopération » entre des acteurs fortement hétérogènes.

Mais, les aspects modélisation en bio-printing sont peu visibles dans les descriptions des articles par leur titre (et également dans la lecture, effectuée par J.C. André, de quelques centaines d'articles qui ont pu paraître pertinents). Visiblement, il s'agit de s'afficher, de « trouver ses marques » en développant des concepts, des méthodes, des procédés, en recherchant des matériaux adaptés et en utilisant des méthodes inductives pour avancer sur ce sujet. L'absence de résultats significatifs en matière de fabrication de vrais tissus effectivement implantables n'autorise pas encore (même si cela constitue une promesse de la part des startups et de certains scientifiques) une vision empreinte de rationalité et de crédibilité opérationnelle.

Dans le fond, les publications (cf. bibliographie) attestent des formes d'induction ou plus prosaïquement de «bricolage savant » dans un domaine en émergence. Indépendamment de ces aspects se posent la question des moyens d'aborder des questions complexes avec des zones de nonconnaissance (cf. figure 7) (l'épistémologie: de la connaissance et de la logique est la branche de la philosophie concernée par la théorie de la connaissance : études de la nature de la connaissance, la rationalité des croyances et sa justification) et de la conduite d'un projet scientifique et technologique. «Ce qui change radicalement, c'est qu'on ne combine plus, au premier temps, les connaissances de chacune des disciplines, mais on commence par rassembler ce qu' on ne sait pas, «The state of the non-art », en quoi la discipline ne saurait donner de réponses aux problèmes que pose l'objet que l'on cherche à connaître et à construire. Chaque discipline cherche en quoi l'objet ne peut être traité par ses concepts et sa logique. Cela interroge au plus profond la pratique scientifique parce que ces non-savoirs obligent à voir l'interdisciplinarité autrement que comme une procédure de somme. Elle obéit à une logique de soustraction (sans manque) qui conduit chaque discipline à être réinterprétée par les autres. La maîtrise n'est plus au centre. Le non-savoir n'est plus à la marge, mais au centre du processus » (Schmid, 2010).
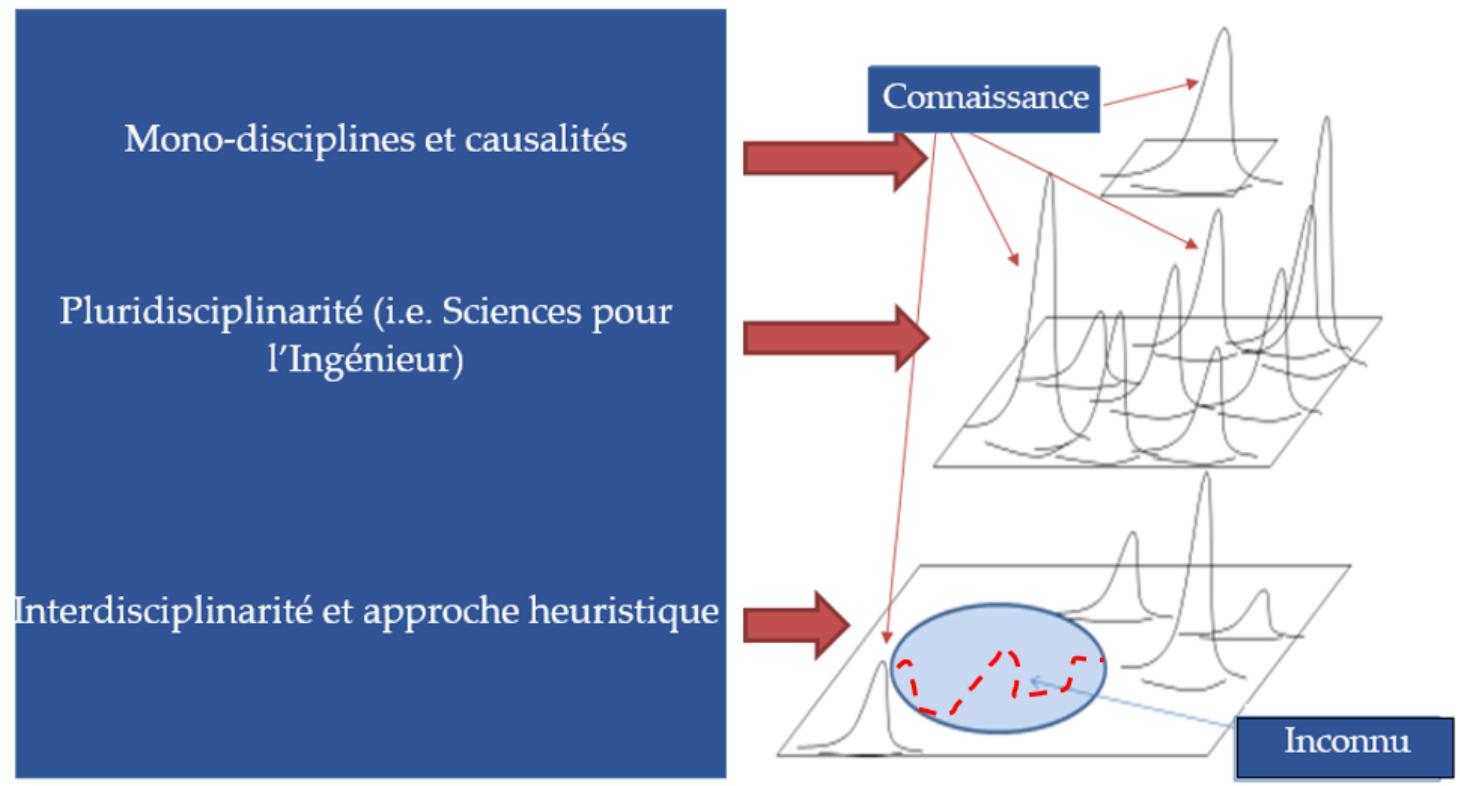

Figure 7. Approches scientifiques : des relations cause-effet à l'abord de la complexité (en pointillés rouge un chemin possible entre causes et conséquences) 
La pratique naturelle des scientifiques est de rechercher si une rigueur mathématique, des procédures algorithmiques peuvent assurer à l'interface entre technologies, sciences de l'ingénieur, sciences de l'information et biologie un corps de doctrine conceptuel qui pourrait assurer une nouvelle connaissance pour la production de tissus et d'organes biologiques (BP), tout en sachant que nul spécialiste ne peut en principe dépasser une culture plus large que celle de sa discipline... Ce travail a été effectué avec la volonté d'associer le maximum de simplicité à une robustesse satisfaisante indispensable pour rendre compte des phénomènes...

Pour le bio-printing, plusieurs disciplines sont à l'œuvre, ce qui amène à « modéliser » des mutualisations de domaines ayant probablement des équations de comportement différents, des interfaces multiples et singulières, des non-linéarités, des changements d'échelles (de composants subcellulaires à l'organe) associés à des formes fractales éventuelles, etc. La complexité envahit les domaines par plusieurs voies: celle des arrangements géométriques, de la complexité de la dynamique (croissance, retrait de l'échafaudage par exemple en BP) et celles de la fonctionnalité, de la robustesse, du coût, etc.

En accord avec Mathieu et Schmid (2014), l'approfondissement peut prendre des formes très variées s'appuyant sur quelques noyaux de connaissances issus des travaux expérimentaux ou sur des objets modèles supports de concepts (mais qui, de toutes les façons, ne trouveront de sens que par une approche interdisciplinaire réellement intégratrice). Cela suppose une décision épistémologique, qui oblige à une réflexion pour le meilleur choix entre différents « discours de la méthode » s'appuyant sur divers piliers : créativité, pensée divergente, sérendipité, exploration de la complexité, preuves de concept, validation expérimentale, modélisation, simulation, etc. Par manque d'échange avec les disciplines convoquées pour le développement du bio-printing, la modélisation mathématique par sa rapidité d'usage (et ses limites) permet de mimer (au moins en partie) les processus impliqués dans la réalisation de bio-construits.

Cet exercice n'a pratiquement pas fait l'objet de l'attention des scientifiques du domaine puisque 10-15 publications environ en font état (cf. par exemple : Kupiec et Sonigo, 2003 ; Kupiec, 2008 ; 2009 ; 2013 ; Ballet et al, 2011 ; Chen et al, 2019 ; Peters et al, 2018 ; Furfaro, 2018 ; Wolpert, Tickle et Arias, 2017 ; Boudon et al, 2015). Lors du développement d'un organisme, les cellules se différencient et les tissus s'auto-organisent spatialement pour former un être vivant complet. Ces phénomènes sont contrôlés par des signaux chimiques ou « morphogènes » capables d'influencer la différenciation d'une cellule. Pour comprendre comment un tissu s'organise, il serait nécessaire de savoir comment les cellules en cours de différenciation décodent l'information. Or, les morphogènes contrôlent l'émergence de modèles spatiaux pendant le développement. Selon Fried et Iber (2015), un gradient morphogènique émerge à travers le champ des cellules. Celles-ci détectent la concentration locale de morphogène et à partir d'un seuil de concentration donné peuvent avoir différents destins. Dans ce qui suit, on « invente » des lois morphogénétiques qui doivent jouer à la fois sur la duplication cellulaire et la différenciation. De même, la prolifération des cellules est contrôlée par des signaux d'activation ou d'inhibition qui elles aussi doivent être «inventées » (par absence de données).

\section{Bases de la modélisation}

D'un point de vue informatique, il est sans doute possible de travailler avec quelques milliers de cellules (Boudon et al, 2015), ce qui représente une dimension de l'ordre de 0.1 à $1 \mathrm{~mm}$, ce qui est loin de représenter un tissu réel, encore moins un organe biologique. Imaginons qu'on soit capable de définir une loi moyenne de comportement pour un ensemble de cellules qui vont constituer un voxel (non vascularisé et non innervé à ce stade). Si ce voxel correspond à un élément représentatif (avec des possibilités de comportements à seuil ou aléatoires), alors il serait envisageable de tenter de gagner sur les échelles spatiales... 
Il sera sans doute possible de supposer pour les besoins de la modélisation, que le système étudié peut être lui-même composé de parties plus grandes en interactions. Ces parties pourront être des entités physiques ou abstraites. Un système sera dit auto-organisateur s'il peut, à partir de ses interactions avec son environnement, détecter toutes les situations non coopératives entre ses parties et tenter de les supprimer en se réorganisant. Un système auto-organisateur est typiquement capable de manière autonome de :

- Créer, supprimer, modifier des parties en son sein ;

- Changer l'organisation (les relations interindividuelles).

Mais, ce qui constitue un élément très important dans ce travail de modélisation, c'est de savoir si l'on peut trouver des conditions ou une vascularisation est susceptible d'apparaître. C'est d'ailleurs, ce que Kupiec $(2008$; 2009 ; 2013) a proposé. Par ailleurs, il serait judicieux de disposer d'un échafaudage initial qui se résorbe en même temps que la vascularisation se met en place. Il y aurait donc simultanéité entre différenciation cellulaire associée à un début de vascularisation et disparition des supports.

\subsection{De la duplication cellulaire à la duplication associée à de la différenciation}

Un modèle de type «automate cellulaire » connexionniste est caractérisé par trois constituants de base : un réseau, une règle d'activation et une règle d'apprentissage (Collectif SMI, 1995 ; Wang et Sun, 2013). Le réseau est composé par un ensemble de cellules placées initialement en un point donné de l'espace (cf. à deux dimensions, l'exemple de la figure 8 dont le principe est issu de Neagu et al, 2006). «La règle d'activation d'un modèle connexionniste est une procédure locale que chaque cellule suit en mettant à jour son niveau d'activation en fonction du contexte d'activation des cellules voisines. Un modèle génétique repose sur l'évolution d'une population dans un environnement selon des critères de survie préfixés, fonction de l'arrivée de nutriments, de l'oxygène, de la présence de cellules mortes, de la possibilité de leur élimination, etc. Le principe général de l'algorithme génétique à développer repose sur un processus continu qui remplace les cellules les moins adaptées (en cours d'apoptose) par d'autres cellules issues d'autres choisies aléatoirement parmi les plus adaptés » (Neagu, 2006).

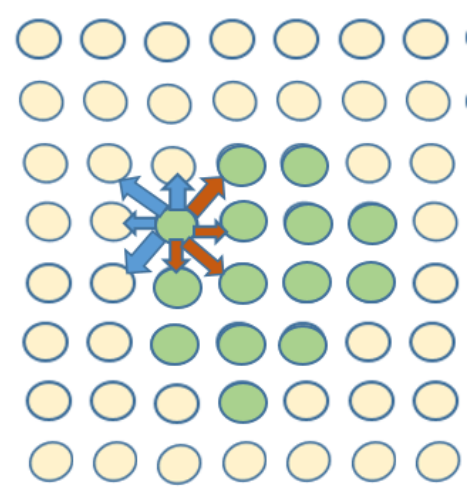

Figure 8. Principe du modèle : chaque cellule interagit avec son environnement pour mourir, proliférer, se déplacer, etc.

Sur cette base, il peut y avoir prolifération cellulaire, migration tant que les critères d'apport de nutriments et d'oxygène sont respectés (McCune et al, 2014; Sun et Zaman, 2017). Après de nombreuses générations, le système doit tendre vers un équilibre dynamique, les cellules présentes (s'il en existe) sont celles qui répondent à la programmation «biologique » recherchée. Tant que les cellules gardent leur nature (pas de différenciation dans cette étape), il doit être possible de démontrer qu'on atteint effectivement un état stationnaire ; de plus, il peut être envisagé de prendre 
en considération l'attractivité éventuelle (ou son contraire) des cellules pour l'échafaudage, favorisant ainsi une migration cellulaire au voisinage du support. Pour autant que les mécanismes d'interaction soient connus, c'est envisageable, mais c'est juste un peu plus compliqué...

Au cours de la dernière décennie, l'avènement des organoïdes a démontré que les cellules ont la capacité de se réorganiser en structures complexes spécifiques aux tissus, compte tenu de facteurs inductifs en faible nombre (Grebenyuk et Ranga, 2019). Toutefois, l'absence d'organisation structurée et de taille raisonnable des bio-construits constitue (encore ?) un obstacle à l'obtention d'une fonctionnalité robuste in vivo. Dans ces conditions, les tissus en développement après bioprinting sont interpénétrés et interagissent avec un réseau complexe de vaisseaux sanguins qui permet non seulement l'échange d'oxygène, de nutriments et de déchets, mais aussi un échange biochimique inductif et un modèle structurel pour la croissance. Dans ce PRD, je mets en œuvre un modèle de réseau dans un algorithme de type «Monte-Carlo» efficace, mais volontairement réducteur, fondé sur des listes pour simuler la fabrication d'un ensemble de constructions de tissus ou d'organes dans plusieurs géométries se rapprochant de cette complexité biologique.

\subsubsection{Duplication simple}

Nous considérons qu'il est possible de déposer dans un espace libre à deux dimensions des cellules de type A qui peuvent se reproduire à condition de disposer d'un flux possible de nutriments. Partant d'une seule cellule, celles-ci se développent pour occuper tout l'espace accessible comme le montre la figure 9 avec un taux de remplissage de l'ordre de 0.36, valeur comprise entre 0 et 1 liée au besoin d'accéder aux nutriments. Le choix du dépôt cellulaire initial n'affecte pas le résultat final.

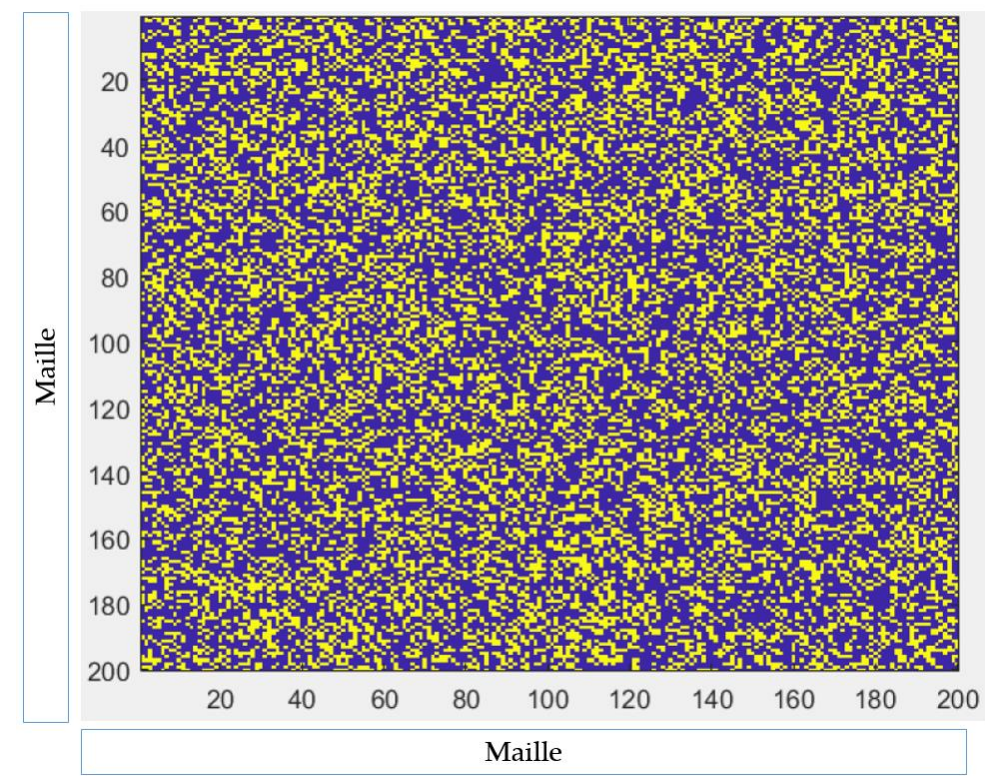

Figure 9. Un exemple de répartition des cellules $A$ dans un espace à 2 dimensions au bout d'un temps où le taux de remplissage est stabilisé

Plusieurs remarques permettent d'aller plus loin dans ce résultat de calcul :

- Compte tenu du grand nombre de cellules à prendre en considération, le choix du langage informatique a été crucial pour atteindre des tendances dans un espace temporel « acceptable» (cf. Annexe). C'est en particulier pour cette raison que l'on s'est limité à un espace à deux dimensions pour gagner en temps calcul. Remarquons que dans le travail préparatoire de Zhong (2019), les tendances entre 2 et 3 dimensions étaient proches, ce qui explique le choix réalisé dans ce travail ; 
- On considère ici que les cellules sont placées dans un milieu pseudo-infini, c'est-à-dire les cellules qui sortent par exemple à gauche de la boite réapparaissaient à droite et inversement (cf. figure 10).

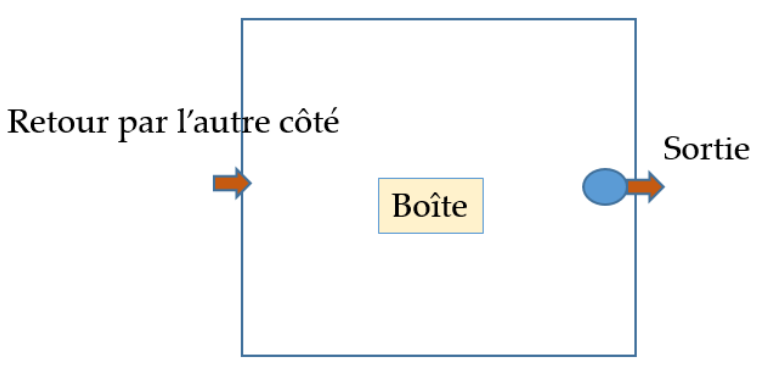

Figure 10. Une cellule qui sort à gauche de la boite réapparaisse à droite

- Ce premier code favorise l'apoptose, ce qui conduit à un taux de remplissage modeste $(0.36$ dans l'exemple de la figure 9. Néanmoins, le principe de la croissance cellulaire (probabilité de mourir, de se dupliquer etc..) sera repris pour dans les paragraphes suivants ;

- Dans cette partie d'initialisation de la modélisation, on considère que chaque cellule A n'interagit qu'avec ses voisins les plus proches avec une probabilité de mourir et/ou de se dupliquer. Les valeurs utilisées pour l'exemple faisant l'objet de la figure 9 font l'objet des données rassemblées dans le tableau 1.

\begin{tabular}{|l|l|l|}
\hline Nombre de voisins & Probabilité de mourir & Probabilité de se dupliquer \\
\hline 0 & $0 \%$ & $100 \%$ \\
\hline 1 & $0 \%$ & $90 \%$ \\
\hline 2 & $10 \%$ & $70 \%$ \\
\hline 3 & $30 \%$ & $50 \%$ \\
\hline 4 & $50 \%$ & $20 \%$ \\
\hline 5 & $80 \%$ & $0 \%$ \\
\hline 6 et + & $100 \%$ & $0 \%$ \\
\hline
\end{tabular}

Tableau 1. Données utilisées dans la modélisation

- Dans ce modèle, on se limite à des interactions de forte proximité, ce qui correspond à une simplification imposée par le besoin d'atteindre des temps calculs non prohibitifs. En situation réelle, les cellules (de taille moyenne de l'ordre de $5 \mu \mathrm{m}$ ) peuvent survivre quand elles sont environnées d'environ 100 cellules dans un espace à deux dimensions (1000 à 3 dimensions). Des organoïdes constitués d'amas cellulaires sont ainsi réalisés pour des applications médicales utilisant des cellules humaines. Ces ensembles permettent différentes évaluations sans qu'il soit nécessaire d'introduire des apports spécifiques de nutriments (cf. par exemple : Seijkens et al, 2018 ; Pasturel, Strale et Studer, 2019).

\subsubsection{Différenciation}

« L'auto-organisation (Fox-Keller, 2009) est un mécanisme ou un ensemble de mécanismes par lesquels des structures sont produites au niveau global d'un système à partir d'interactions entre ses constituants à un niveau d'intégration inférieur. Les interactions entre constituants sont elles-mêmes produites localement sans aucune référence à une structure globale préconçue. Au contraire, c'est une propriété émergente du système et non une propriété imposée de l'extérieur au système ». Cette définition amène à introduire le concept d'émergence de structures méta, voire globales, à partir 
d'interactions entre constituants « élémentaires » à l'échelle locale. Il peut s'agir d'autoorganisations de structures et/ou de fonctions.

Jusqu'ici, on a tenté de réfléchir à un assemblage spontané de cellules identiques (sans sélection naturelle). Il est possible que sous l'influence de l'environnement, des cellules issues de cellules souches (en gris clair sur la figure 11 haut) se différencient en cellules A ou B. Plusieurs occurrences peuvent sans doute exister : prolifération des A relativement aux B (et réciproquement) ou une prolifération coordonnée (exemple des vaisseaux sanguins qui se construisent en même temps que le tissu fonctionnel). Selon les types d'interaction entre les cellules A et les B, Kupiec (2008) a réalisé différentes simulations qui conduisent à des résultats qu'il nous a paru utile d'approfondir pour juger de leur utilité pour le bio-printing. Les figures 14 illustrent quelques résultats présentés dans son livre et dans Kupiec (2009). Des résultats similaires ont été obtenus à la même époque par Merks et Koolwijk (2009). En effet, une question très importante est soulevée dans nombre de travaux sur le bio-printing, celui du transfert de matière et d'énergie chimique vers les cellules imprimées (Merks et Koolwijk, 2009). Comment, dans une étape importante modéliser l'angiogenèse ? Même si des raffinements existent (Okuda et al, 2016; Sun et Zaman, 2017), un approfondissement dans ce type de simulation - fortement réducteur - relié à l'expertise de collègues biologistes pourrait être susceptible d'expliquer un potentiel de différenciation cellulaire... conduisant à l'apparition de micro-vaisseaux ; quand le mécanisme sera compris (et surtout validé expérimentalement), il faudra rechercher les conditions pour que l'on maitrise correctement cette évolution indispensable du tissu bio-imprimé. Cette compétence à acquérir constitue un élément essentiel qui devra se trouver dans la boîte à outils de tout bon bioimprimeur... même si cela ne fait pas tout...
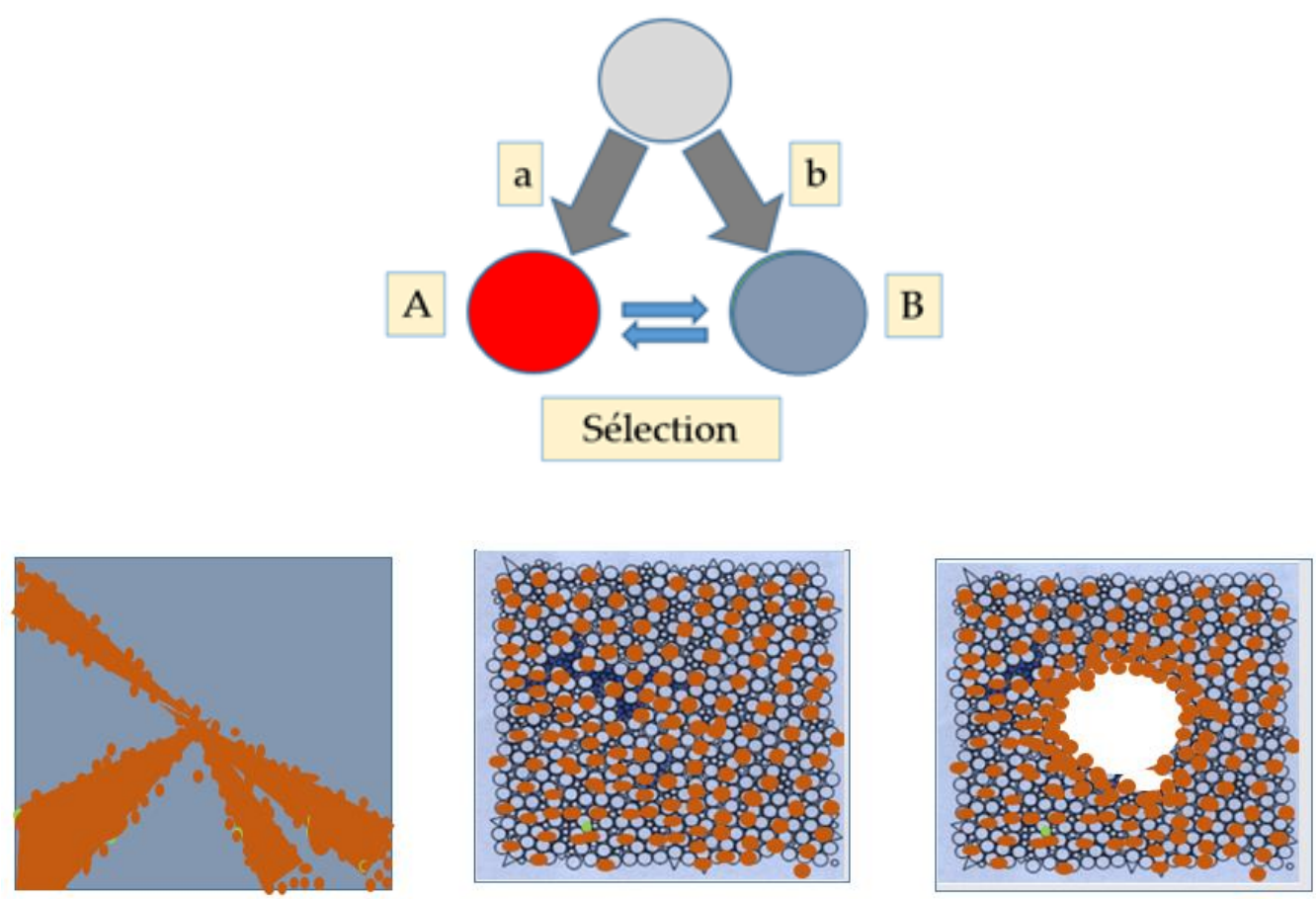

Différentes formes d'équilibre sélectif selon la valeur des paramètres d'influence entre A et $\mathrm{B}$ : Zones séparées ; Répartition homogène ; Formation de lacunes ; etc.

Figure 11. Dessins approximatifs de simulations effectuées par Kupiec (2008) et par Merks et Koolwijk (2009). 


\section{Remarque 1 : Bio-construit à partir de deux types de cellules}

Kim et al (2020) pour réaliser par bio-printing un muscle qui imite les caractéristiques structurelles et fonctionnelles du muscle natif ont étudié les effets de l'intégration de cellules neurales dans la construction musculaire squelettique bioimprimée pour accélérer la régénération musculaire fonctionnelle in vivo. L'apport neural indique une amélioration dans la formation de myofibres, la survie à long terme et la formation de jonction neuromusculaire in vitro. Plus important encore, les constructions bioimprimées avec intégration des cellules neurales faciliteraient une innervation rapide. Cette méthode qui est encore à approfondir n'est pas celle qui a été suivie ici, mais présente une voie alternative intéressante.

\section{Remarque 2 : notion d'ordre - Fonction de redondance $\mathbf{R}$ de Shannon}

Un système biologique sera dit complexe s'il présente des phénomènes d'émergence, c'est-à-dire qu'il a une richesse potentielle supérieure à celle de la somme des sous-systèmes qui le composent. Cette situation peut être décrite par l'inéquation suivante :

$$
\mathrm{H}(\mathrm{x}, \mathrm{y})>\mathrm{H}(\mathrm{x})+\mathrm{H}(\mathrm{y})
$$

avec $\mathrm{x}$ et $\mathrm{y}$ des variables de deux sous-systèmes et $\mathrm{H}$ une fonction mathématique qui exprime la richesse potentielle d'un système (en parlant de richesse, on fait apparaître un concept appréciatif discuté plus loin). Cette fonction est appelée en physique statistique l'entropie du système (Ben Amor, Lavallée et Bui, 2006). Les systèmes complexes présentent en général un certain nombre de caractéristiques qui font qu'ils sont plus difficiles à comprendre et à gérer que les systèmes simples et compliqués (Gallopin et al, 2001) :

- Multiplicité des points de vue légitimes : Par exemple, il est difficile de comprendre un système adaptatif sans en considérer aussi le contexte et sans tenir compte des " points de vue » et des intérêts des différents éléments en cause, en particulier vus depuis différentes disciplines (aucune ne détenant probablement la « vérité » à elle-seule). Mais la recherche de systèmes de prise d'information est dans ce contexte indispensable, les milieux vivants de type agrégats de cellules sont relativement muets ;

- Non-linéarité : Les systèmes complexes ne sont pas linéaires, en ce sens que de nombreuses relations entre leurs éléments ne le sont pas, de sorte que l'ampleur des effets n'est pas proportionnelle à celle des causes et que le répertoire des comportements est très riche (comportement chaotique, multi-stabilité en raison de l'existence de plusieurs états d'équilibre et de processus de régulation, processus de dérapage, etc.). La non-linéarité joue un rôle décisif dans la naissance de comportements contre-intuitifs typiques de nombreux systèmes complexes ;

- Emergence : Cette propriété systémique, qu'exprime la phrase « le tout ne se réduit pas à la somme des parties », signifie que l'on ne peut comprendre les propriétés des parties que dans le contexte plus large de l'ensemble et que l'analyse (sans résidu) de celui-ci n'est pas réductible à celle de ses parties. Un élément réellement nouveau peut surgir de l'interaction (non-linéaire ?) des éléments du système ;

- Auto-organisation : Phénomène par lequel des éléments inter-agissants coopèrent pour produire un comportement et des structures coordonnées à grande échelle (telles que les configurations créées par les structures dissipatives). L'apparition d'une structure de plus haut niveau est produite sans la nécessité d'un principe organisateur en faisant preuve d'une certaine autonomie et de robustesse (Di Marzo Serugendo, Gleizes et Karageorgos, 2006 ; Ottino, 2004 ; Fromm, 2004 ; Bar-Yam, 2003) ; rappelons que pour Asby, (cité par Jacob, 1970), l'auto-organisation est normalement impossible : « un système ne peut pas s'auto- 
organiser, car cela voudrait dire changer sa loi d'organisation suivant une autre loi qui devrait être elle-même son organisation... »; c'est un autre débat...;

- Multiplicité des types de complexité : combinatoire entre cellules entre elles d'une part, et entre cellules et échafaudage d'autre part ; morphologique ; fonctionnelle ;

- Multiplicité des échelles : Beaucoup de systèmes complexes sont hiérarchiques, en ce sens que chaque élément du système est un sous-système d'un système d'ordre inférieur, et le système lui-même est un sous-système d'un « supra-système » d'ordre supérieur. Ce qui est important, c'est que dans beaucoup de systèmes complexes, il y a un puissant couplage entre les différents niveaux, de sorte que le système doit être analysé ou géré à plusieurs échelles à la fois. Cependant, les systèmes à différents niveaux d'échelle ont différentes sortes d'interactions et aussi différents rythmes caractéristiques d'évolution. Il est donc impossible d'avoir à propos d'un système un point de vue unique, juste et exhaustif, même à un seul niveau de système. Pluralité et incertitude sont inhérentes au comportement des systèmes complexes ;

- Incertitude irréductible : Il y a, dans les systèmes complexes, de nombreuses sources d'incertitude. Certaines sont réductibles moyennant un complément de données et de recherches, notamment lorsque l'incertitude est due à des processus aléatoires (se prêtant à une analyse statistique ou probabiliste, pour autant que cela soit adapté à des expérimentations reproductibles avec des paramètres contrôlés) ou à l'ignorance (absence de données ou ensembles de données inadéquats, définition incomplète du système et de ses limites, compréhension incomplète ou mauvaise compréhension du système).

Bien que l'on puisse trouver dans certains systèmes compliqués, voire simples, quelques-unes de ces caractéristiques (notamment la non-linéarité ou l'incertitude), le fait est qu'un système complexe peut les posséder toutes... (Pavé, 2012). R est défini par

$$
\mathrm{R}=1-\mathrm{H} / \mathrm{Hmax}
$$

où Hmax est la quantité d'information qui correspond à un système de diversité en l'absence de redondance. L'organisation jouerait sur une sorte de compromis entre la quantité d'information et la redondance... L'auto-organisation serait associée à une augmentation de la quantité d'information (programmation interne ? (cf. par exemple Kaern et al, 2006)). Pour Rothstein (1962), l'ordre du système serait défini par R.Hmax R étant dépendant des contraintes du système et Hmax de la diversité potentielle dudit système (cf. également Atlan, 2006).

Sous l'influence de perturbations, pour des valeurs de $\mathrm{R}$ élevées, le système peut continuer à fonctionner tant qu'une certaine redondance peut remédier aux pertes de communication selon le principe d'ordre par le bruit (Von Foerster, 1960). Ce principe dans lequel les niveaux d'intégration sont emboîtés les uns dans les autres pose la question de la qualité de l'information (signification et transmission locale). Atlan (2006) prend l'exemple d'une cellule affectée par un « bruit » qui la désorganise, ce qui l'amène à se diversifier relativement à ses voisines traduite à un niveau plus élevé par une création de variété et d'adaptation possibles... avec de possibles feedbacks. En présence de bruit, le système le décode et peut élaborer de nouveaux comportements.

Le modèle de Shannon constitue un schéma à partir duquel l'information permet à l'organisation d'adapter son comportement à chaque instant par régulation, se transformer, se rééquilibrer afin d'être en cohérence thermodynamique avec l'environnement. L'information donne lieu à un processus d'ajustement permanent de l'organisation par les canaux (le système s'adapte par accommodation) et les codes (le système s'adapte par assimilation) de communication par rapport à un projet. 
Shannon dissocie quantité et signification de l'information : la quantité d'information contenue dans un message peut être mesurée indépendamment de toute référence au sens de ce message. Appliquée à la biologie, elle implique que la quantité d'information portée par un brin d'ADN, d'une organelle, d'une cellule, etc. ne dépend pas de la connaissance fine de l'organisme dont la construction, le développement et la sénescence sont programmés, au moins en partie, par cet ADN. Cette idée, derrière les différences apparentes de comportement, des structures qui les relient, pose la question de savoir si ces différences cachent au fond le même phénomène. Le débat est à trancher (Frank et Gouyon, 1997).

D'un point de vue pratique, ce qui est recherché c'est la possibilitéde partir de cellules A déposées initialement, d'observer en même temps que la production de cellules $\mathrm{A}$ et $\mathrm{B}$ leur séparation (création d'un ordre défini semi-qualitativement). Le paramètre $\mathrm{R}$, compris entre 0 (répartition aléatoire des A et des B) et 1 (cellules de même types regroupées) a été calculé de la façon suivante :

$$
\mathrm{R}=\mid \text { voisin } A-\text { voisin } B \pm 1 \mid / \text { total_voisin (le } \pm 1 \text { dépend si la cellule est un A ou un B) }
$$

\subsubsection{Conditions semblables d'échange et de croissance des A et des B}

On considère toujours que les interactions entre les cellules se font avec leur 8 (maximum) cellules proches. Quand les conditions d'échange A/B sont semblables, partant d'une seule cellule A, la figure 12 met en évidence une faible séparation entre les A et les B. Dans les conditions de la figure, $\mathrm{R}$ est de l'ordre de 0.6. Cette valeur est liée à l'effet de l'environnement d'une cellule, alors que la transition $\mathrm{A}=>\mathrm{B}$ est identique à la transition $\mathrm{B}=>\mathrm{A}$.

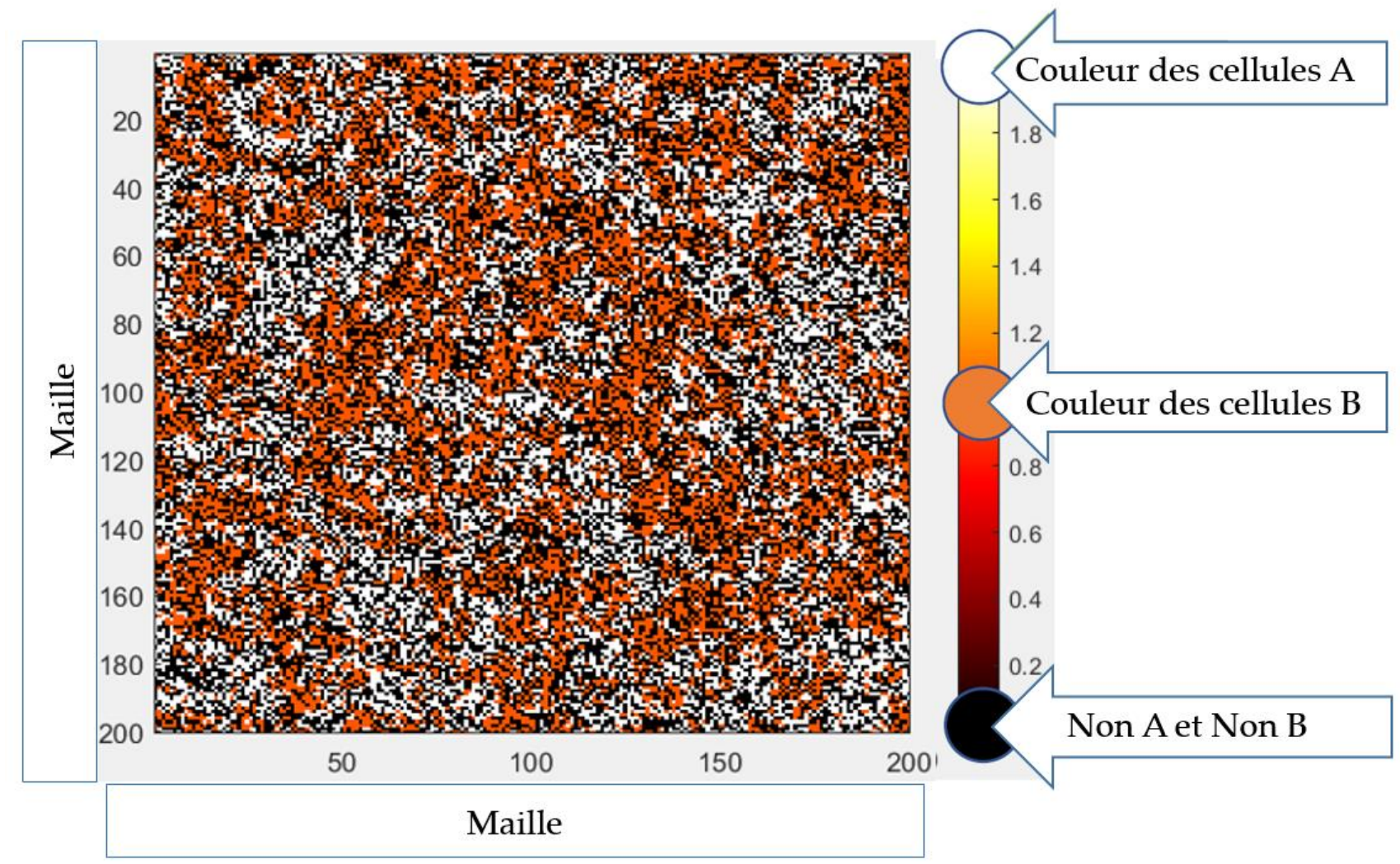

Figure 12. Répartition des $A$ et des $B$ au temps longs 
Les tableaux 2 et 3 rassemblent les données utilisées pour cette modélisation.

\begin{tabular}{|l|l|l|}
\hline Nombre de voisins & Probabilité de mourir & Probabilité de se dupliquer \\
\hline 0 & $0 \%$ & $100 \%$ \\
\hline 1 & $0 \%$ & $100 \%$ \\
\hline 2 & $0 \%$ & $80 \%$ \\
\hline 3 & $0 \%$ & $60 \%$ \\
\hline 4 & $15 \%$ & $40 \%$ \\
\hline 5 & $35 \%$ & $20 \%$ \\
\hline 6 & $55 \%$ & $10 \%$ \\
\hline 7 & $75 \%$ & $0 \%$ \\
\hline 8 & $100 \%$ & $0 \%$ \\
\hline
\end{tabular}

Tableau 2. Données utilisées dans la modélisation (Identité de comportement des $A$ et des $B$ )

\begin{tabular}{|c|c|c|c|c|c|c|c|c|c|c|}
\hline & & \multicolumn{9}{|c|}{ Nombre de voisins du même type } \\
\hline & & 0 & 1 & 2 & 3 & 4 & 5 & 6 & 7 & 8 \\
\hline \multirow{9}{*}{ 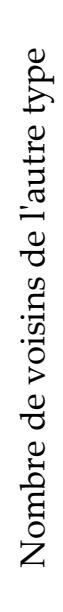 } & 0 & $10 \%$ & $10 \%$ & $5 \%$ & $5 \%$ & $0 \%$ & $0 \%$ & $0 \%$ & $0 \%$ & $0 \%$ \\
\hline & 1 & $20 \%$ & $15 \%$ & $10 \%$ & $5 \%$ & $5 \%$ & $0 \%$ & $0 \%$ & $0 \%$ & \\
\hline & 2 & $30 \%$ & $25 \%$ & $20 \%$ & $15 \%$ & $10 \%$ & $5 \%$ & $0 \%$ & & \\
\hline & 3 & $40 \%$ & $30 \%$ & $25 \%$ & $15 \%$ & $10 \%$ & $5 \%$ & & & \\
\hline & 4 & $50 \%$ & $40 \%$ & $25 \%$ & $20 \%$ & $10 \%$ & & & & \\
\hline & 5 & $50 \%$ & $40 \%$ & $30 \%$ & $20 \%$ & & & & & \\
\hline & 6 & $70 \%$ & $50 \%$ & $40 \%$ & & & & & & \\
\hline & 7 & $80 \%$ & $70 \%$ & & & & & & & \\
\hline & 8 & $85 \%$ & & & & & & & & \\
\hline
\end{tabular}

Tableau 3. Probabilité de se transformer en une cellule de l'autre type

\subsubsection{Conditions d'échange et de croissance des $A$ et des $B$ favorisant leur séparation}

Dans l'étape présentée ci-dessus, en donnant aux A et aux B des comportements identiques, il n'est pas envisageable d'espérer une séparation spatiale des deux espèces cellulaires. Pour atteindre cet objectif, il faut privilégier le comportement d'une des deux espèces relativement à l'autre. Ainsi, il est envisageable de favoriser la duplication des A par rapport aux B (une plus grande probabilité de se dupliquer pour A que pour B), par exemple si la cellule possède plus de 2 voisins. En maintenant les paramètres comme précédemment, la simulation favorisera simplement le développement des $\mathrm{A}$ par rapport aux $\mathrm{B}$, et il y aura plus de $\mathrm{A}$ que de $\mathrm{B}$ à la fin. Mais, d'un autre côté, si les A ont plus de chance de se transformer en B que l'inverse ou que les B ont une plus faible probabilité de se transformer en A (par exemple s'ils possèdent plus de 3 voisins de type B), cela aura pour effet de créer des blocs de B comme le montre la figure 13 (avec un paramètre d'ordre d'environ 0.9). Dans les deux exemples présentés, il ne semble pas envisageable de dégager des formes apparentes de déterminisme spatial. Cette situation pourrait signifier une difficulté de positionner à l'avance les zones où pourraient apparaître des nerfs ou des micro-vaisseaux... Ce que l'on peut constater à l'examen de ces figures, c'est que si des cellules B apparaissent en début de calcul, elles ont plus tendance à recouvrir une très grande surface. Dans ces conditions, les cellules A n'auront d'autre choix que d'occuper l'espace libre non encore ouvert par les B pour se 
développer (ce que l'on observe sur la seconde simulation). Au contraire, si les B apparaissent plus tard, ils formeront des agrégats plus petits avec des cellules A qui comblent les interstices (C'est ce que l'on observe sur la première simulation). Il n'est pas possible de conclure que le résultat est sensible aux conditions initiales, mais que l'histoire du bio-construit a un effet sur le résultat au temps (de calcul) longs.

Une autre chose prévisible mais que l'on peut néanmoins constater, c'est que on observe souvent des petits paquets de $\mathrm{B}$ à l'intérieur d'une masse de $\mathrm{A}$ (car les $\mathrm{B}$ ont été conçus comme assez résistants face à leur transformation en $\mathrm{A}$ ), alors qu'on observe relativement peu de petits paquets de A à l'intérieur de groupes de $\mathrm{B}$ (puisque ceux-ci sont moins résistants).

Cette situation menée par essais et erreurs indique une telle possibilité. Sans doute est-il possible d'en trouver d'autres tout aussi crédibles. Les valeurs utilisées pour cette simulation sont rassemblées dans les tableaux 4 à 6.

\begin{tabular}{|c|c|c|c|}
\hline Nombre de voisins & Probabilité de mourir & $\begin{array}{c}\text { Probabilité de se } \\
\text { dupliquer pour A }\end{array}$ & $\begin{array}{c}\text { Probabilité de se } \\
\text { dupliquer pour B }\end{array}$ \\
\hline 0 & $0 \%$ & $100 \%$ & $100 \%$ \\
\hline 1 & $0 \%$ & $100 \%$ & $100 \%$ \\
\hline 2 & $0 \%$ & $90 \%$ & $80 \%$ \\
\hline 3 & $0 \%$ & $80 \%$ & $60 \%$ \\
\hline 4 & $15 \%$ & $60 \%$ & $40 \%$ \\
\hline 5 & $35 \%$ & $50 \%$ & $20 \%$ \\
\hline 6 & $55 \%$ & $30 \%$ & $10 \%$ \\
\hline 7 & $75 \%$ & $10 \%$ & $0 \%$ \\
\hline 8 & $100 \%$ & $0 \%$ & $0 \%$ \\
\hline
\end{tabular}

Tableau 4. Probabilité de mourir et de se dupliquer en fonction du nombre de voisins

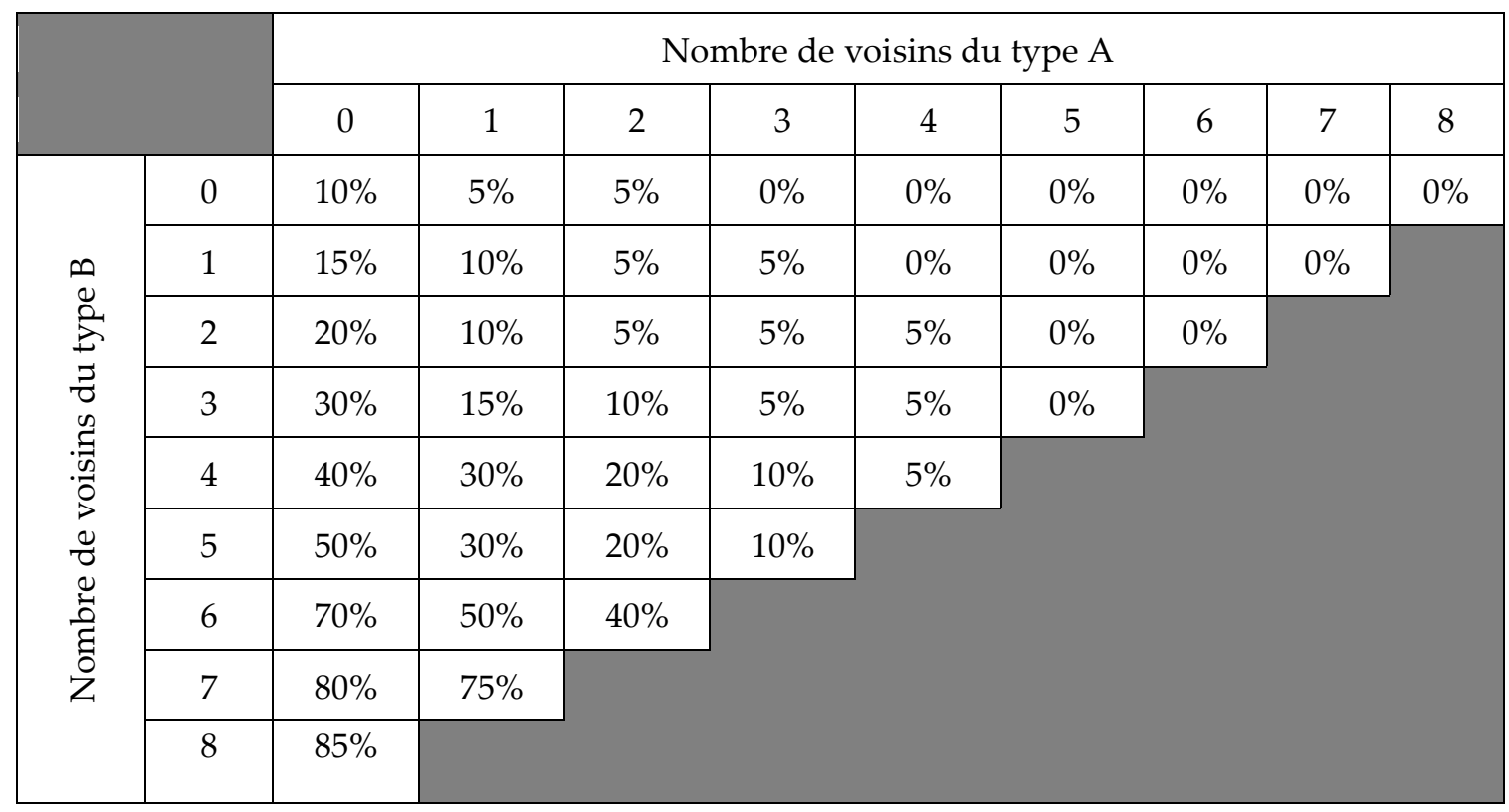

Tableau 5. Probabilité de transformer des $A$ en $B$ 


\begin{tabular}{|c|c|c|c|c|c|c|c|c|c|c|}
\hline & & \multicolumn{9}{|c|}{ Nombre de voisins du type B } \\
\hline & & 0 & 1 & 2 & 3 & 4 & 5 & 6 & 7 & 8 \\
\hline \multirow{9}{*}{ 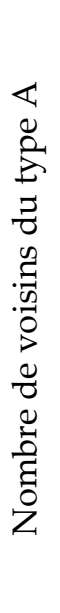 } & 0 & $0 \%$ & $0 \%$ & $0 \%$ & $0 \%$ & $0 \%$ & $0 \%$ & $0 \%$ & $0 \%$ & $0 \%$ \\
\hline & 1 & $5 \%$ & $0 \%$ & $0 \%$ & $0 \%$ & $0 \%$ & $0 \%$ & $0 \%$ & $0 \%$ & \\
\hline & 2 & $7.5 \%$ & $5 \%$ & $2.5 \%$ & $0 \%$ & $0 \%$ & $0 \%$ & $0 \%$ & & \\
\hline & 3 & $10 \%$ & $10 \%$ & $5 \%$ & $5 \%$ & $0 \%$ & $0 \%$ & & & \\
\hline & 4 & $15 \%$ & $15 \%$ & $10 \%$ & $5 \%$ & $0 \%$ & & & & \\
\hline & 5 & $25 \%$ & $20 \%$ & $15 \%$ & $5 \%$ & & & & & \\
\hline & 6 & $30 \%$ & $25 \%$ & $20 \%$ & & & & & & \\
\hline & 7 & $35 \%$ & $30 \%$ & & & & & & & \\
\hline & 8 & $40 \%$ & & & & & & & & \\
\hline
\end{tabular}

Tableau 6. Probabilité de transformer des $B$ en $A$
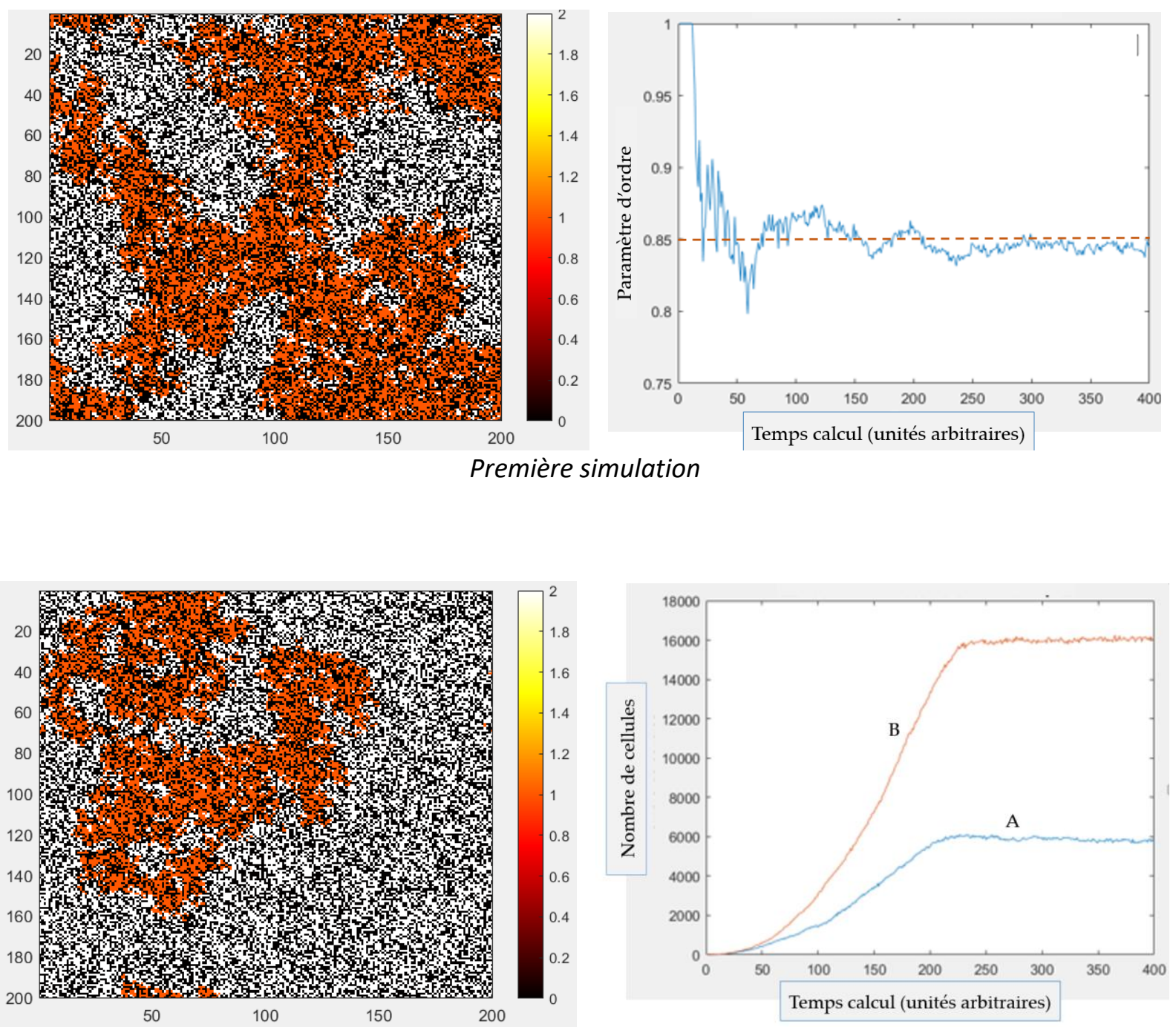

Seconde simulation

Figure 13. Illustration de la possibilité de séparer spatialement les $A$ et les $B$ 


\subsubsection{Vers une vascularisation?}

Dans les conditions présentées figure 13, il n'est pas possible de faire apparaître des zones favorables (à deux dimensions) à la création possible de vaisseaux ou de micro-vaisseaux comme cela a été proposé par Kupiec (2008). Pour atteindre un tel objectif, il a fallu ajouter une contrainte supplémentaire au système en évolution: faire intervenir (ce qui est connu en biologie) des interactions à des distances supérieures aux simples distances cellulaires.

Ainsi, la différence majeure avec la simulation précédente, c'est qu'on suppose que les B ne peuvent pas survivre s'ils se situent à une distance de plus de $\alpha$ cases d'un $\mathrm{A}$ (où $\alpha$ est un entier). Le choix de $\alpha$ influe beaucoup sur l'évolution des cellules comme cela est montré ci-après. Les matrices de probabilités de transformation des $\mathrm{B}$ en $\mathrm{A}$ et des $\mathrm{A}$ en $\mathrm{B}$ sont les mêmes que précédemment (cf. tableau 3).

La figure 14 réalisée pour $\alpha=5$ met en évidence l'apparition d'espaces libres qui pourraient être à l'origine de possibles vaisseaux. Remarquons que, sur cette figure, ce sont les cellules A qui limitent l'espace vide. Dans ce calcul, une cellule de B meurt si elle est située à une distance supérieure à $\alpha$ d'une cellule $\mathrm{A}$. En dessous, les règles d'évolution sont inchangées.

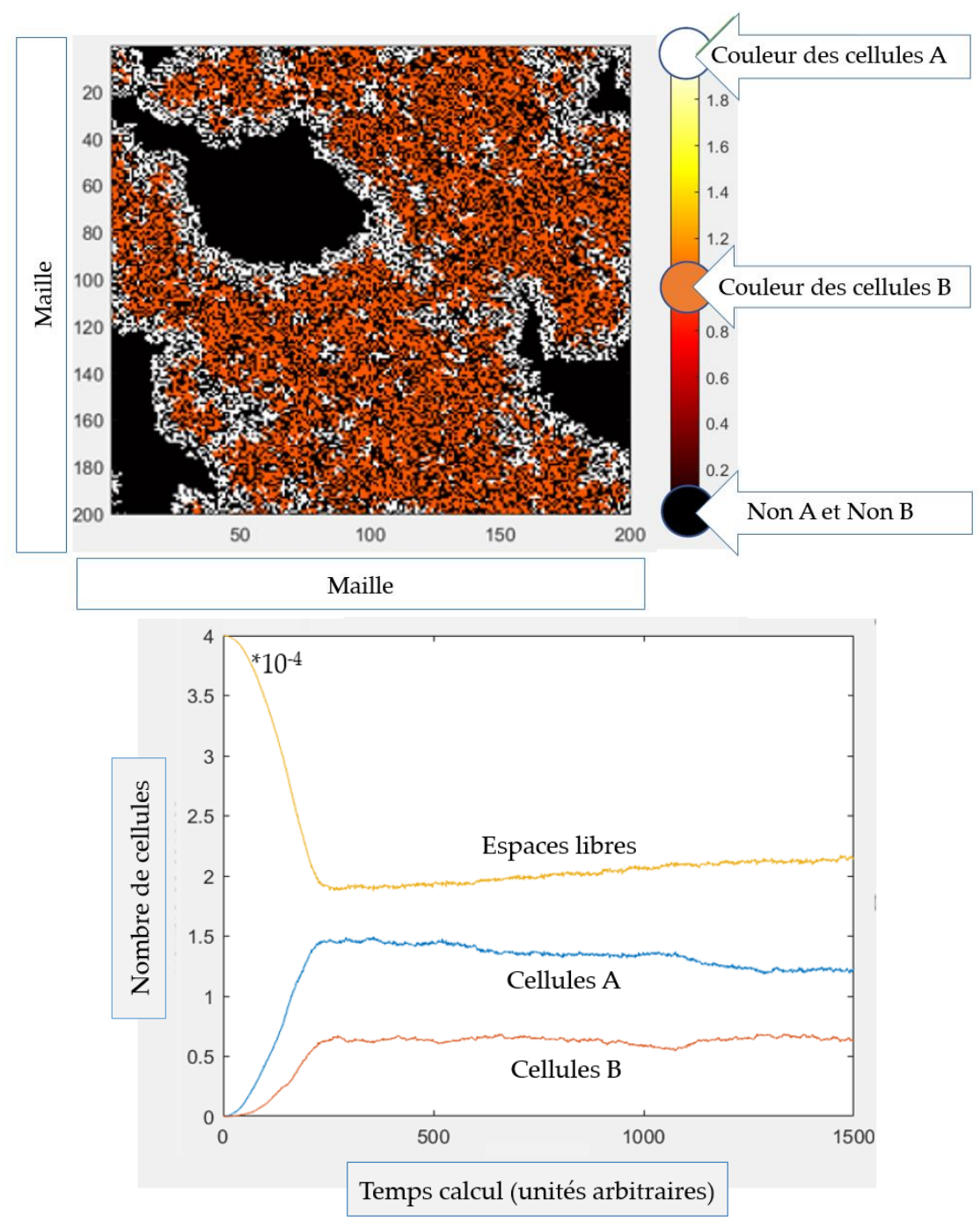

Figure 14. Cas où $\alpha=5$

Si $\alpha$ est supérieur est ou égal à 4, les cellules se développent, puis finissent par disparaître. En fait, les B occupent de plus en plus d'espace et créent des trous de plus en plus gros. La population des $\mathrm{A}$ se réduit jusqu'à leur disparition. Or, dans la simulation, les $\mathrm{B}$ doivent être positionnés à une distance d'au moins $\alpha^{*}$ taille cellulaire pour survivre, ce qui conduit sur le long terme à la 
disparition des B. Le problème est que les B détruisent des A pour disposer de la place conduisant à l'émergence de trous, mais, dans le compromis recherché, si on augmente la résistance des A, les B n'auront plus assez d'espace pour former des trous...

Néanmoins, ce que montrent ces simulations, c'est qu'avec un nombre modeste de paramètres, croissance et différenciations cellulaires peuvent participer (virtuellement) à ce qui pourrait ressembler à un tissu biologique.

\section{Remarque 1 :}

Si on choisit $\alpha$ plus petit que 4, l'épaisseur de « confort » des B est donc plus faible ; alors, les A ont une probabilité non nulle de traverser la barrière des B formant les trous et finissent par combler ceux-ci. Dans ce cas, le résultat de la simulation est très similaire à celui présenté figure 13 (même si on peut observer le début de formation de trous).

Deux distances d'interactions sont à prendre en considération. Les interactions de proximité situées à la distance intercellulaire (comme dans les simulations précédentes) caractérisent l'évolution classique. Une seconde distance d'interaction visant la disparition des B pour créer des espaces libres, qui est variable (cf. paramètre $\alpha$ ).

Or, on sait que les interactions primaires ne produisent déjà à des distances supérieures à la distance intercellulaire. Par simplicité et volonté de réduire le temps calcul, ces effets n'ont pas été pris en compte: il faut rappeler que les propriétés des cellules dépendent du nombre de voisin (probabilité de mourir, de se dupliquer, de se transformer,...). Avec une distance d'interaction de 1, cela conduit à 8 voisins. Sachant qu'il y a deux types de cellules, cela conduit à des matrices de probabilité de $9 \mathrm{x} 9$ (de 0 à 8 voisins de chaque type). Si l'on considère simplement les voisins à 3 distances intercellulaires, cela correspond à un total de 48 voisins, soit des matrices de taille $49 \times 49=2401$. Le nombre de voisins suit une relation de la forme :

$$
n b_{\text {voisin }}=(\text { distance } * 2+1)^{2}-1
$$

Cela devient très vite très consommateur de renseigner autant de probabilités (choisies arbitrairement, mais en faisant en sorte qu'elles aient un peu de sens). Ce qui a été testé, c'est de regrouper le nombre de voisins en palier. Par exemple, de 0 à 5 voisins, une valeur, de 6 à 10 une autre etc... Cela conduit à des résultats proches avec les deux premières simulations. Cependant, on observe que le paramètre d'ordre diminue, car les proches voisins ont autant d'influence que ceux qui sont plus lointains. Par exemple ici, la cellule blanche centrale devrait mourir ou se transformer car entourée de cellules rouges. Mais en comptant ses voisins à 3 distances intercellulaires, elle a plus de voisins blancs que rouges. Elle va donc pouvoir survivre sans problèmes alors qu'initialement ce n'était pas possible. De plus, si elle est située à une distance d'interaction trop élevée, cela revient à dire que toutes les cellules sont directement voisines les unes des autres, ce qui n'a pas vraiment d'intérêt. Il se trouve donc que principe de simplicité et physique du système vont dans le même sens ! (cf. figure 15). 


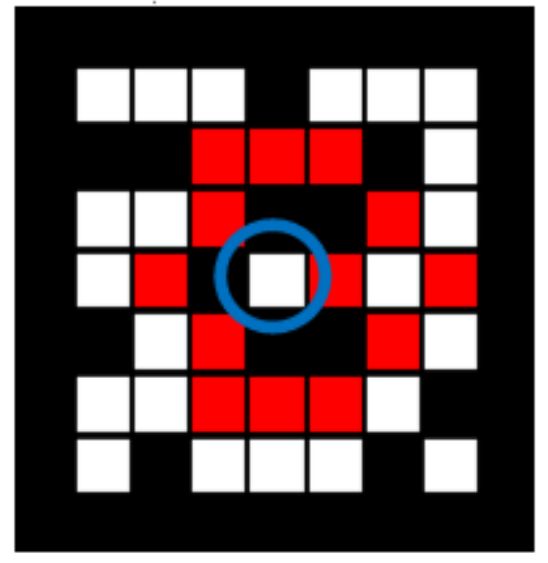

Figure 15. La question des interactions à longue distance

\section{Remarque 2 :}

Un changement de comportement par rapport à la situation présentée figure 14 peut être observé quand la probabilité de transformation des $\mathrm{A}$ en $\mathrm{B}$ a été réduite pour éviter que les $\mathrm{B}$ ne détruisent à terme tous les A. La figure 16 présnte un résultat avec des tailles d'espaces libres plus modestes que dans le cas présenté figure 14.

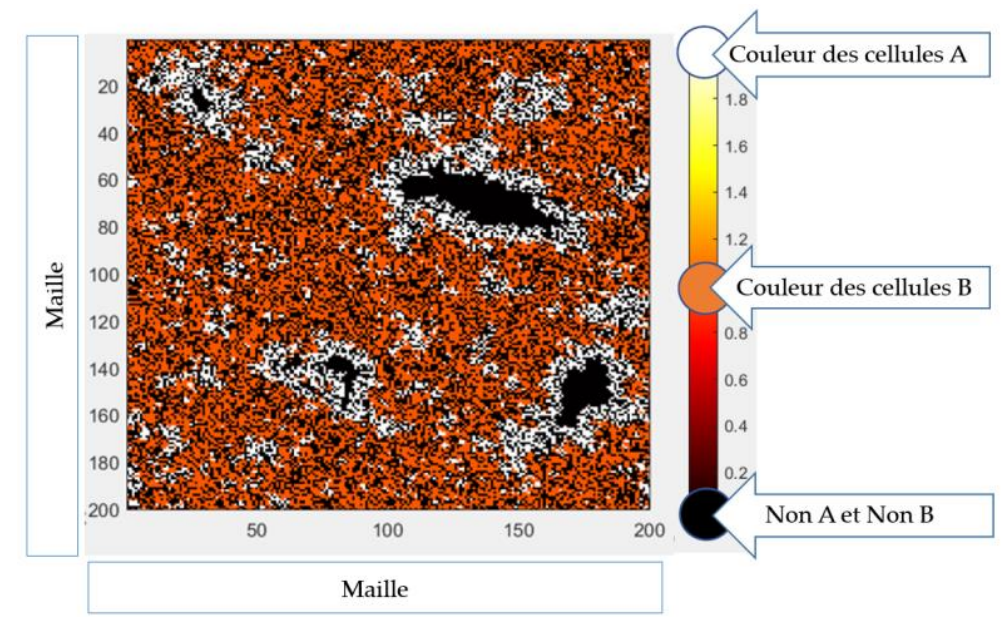

Figure 16. Autre exemple de création d'espaces libres entre les cellules $A$ et les $B$

\subsection{Importance des échafaudages dans la différenciation}

Pour que les cellules survivent, il a été rappelé qu'il pouvait être avantageux de déposer des cellules sur un échafaudage de manière à ce qu'elles puissent être alimentées en nutriments. Ce n'est d'ailleurs pas la seule technique envisageable puisque a proposé des dépôts cellulaires sur des conduits transportant les nutriments (cf. par exemple, Grigoryan et al, 2019 ; Skylar-Scott et al, 2019 ; Balthazar et al, 2019 ; Ozbolat et Hospodiuk, 2016 ; Skardal et Atala, 2015). Le transfert de ces derniers au travers de la membrane constituant l'échafaudage permet l'alimentation cellulaire (toutefois, rien n'est dit sur les performances hydrodynamiques de tels systèmes d'irrigation, ni sur leur devenir). Selon Jessus et al (2017), « la morphogénèse [...] est un processus d'auto-organisation de cellules qui grandissent, se divisent, se déplacent, ou meurent en fonction des événements auxquelles elles ont été soumises dans le passé, de leur « histoire familiale » et des communications qu'elles établissent avec leurs voisines $» . .$. Bref, on est bien loin de la fabrication additive traditionnelle... Pour enfoncer le clou, elle écrit également : «Certaines cellules animales cultivées sur des gels adoptent des destins différents selon la rigidité mécanique du gel : elles deviennent des cellules de cerveau sur un gel mou, d'os sur un gel dur ou de muscle sur un gel de rigidité 
intermédiaire. [...] En réponse aux forces qui leur sont appliquées, les cellules réorganisent leur squelette interne pour mieux résister à ces forces, pour changer de forme ou pour orienter leur prochaine division $» .$. . (Voir également Landau et al, 2018). S'il en est ainsi, la nature et la forme du support des cellules peut donc avoir une grande importance sur la structure du bio-construit puisque le support peut disposer d'un rôle dans la différenciation, au moins pendant qu'il existe (rappelons que sa mission est provisoire, puisqu'il doit être résorbable).

Par ailleurs, selon Chen et al (2019), les cellules sont sensibles à la géométrie de leur environnement et en particulier de la courbure du milieu sur lequel elles évoluent (du nanomètre à plusieurs dizaines de $\mu \mathrm{m}$ ). Selon ces auteurs, la migration des cellules repose sur leur cytosquelette qui leur donne leurs propriétés mécaniques. Les résultats de cette équipe, éloignée du bio-printing, mettent en lumière la manière dont les cellules perçoivent la forme de leur environnement et permettent de mieux comprendre les modalités de la migration cellulaire, impliquée dans de nombreux phénomènes biologiques fondamentaux, tels que la formation des organes, la cicatrisation ou la propagation de tumeurs cancéreuses. Cependant, compte tenu de la structure de la maille utilisée dans les simulations, les effets de courbure n'ont pas pu être pris en considération.

\subsubsection{Echafaudage non biodégradable}

Par souci de faire émerger des situations extrêmes, dans une première hypothèse, il est considéré que les A sont détruits au contact de l'échafaudage à la différence des B. Relativement aux cas précédents, le milieu est borné soit de manière permanente (ce cas), soit de manière temporaire (si l'on fait intervenir une dégradation; cf. §3.2.2). Avec des bords non destructibles, compte tenu de l'hypothèse où les $\mathrm{A}$ ne peuvent exister au contact avec l'échafaudage, il est normal d'attendre que seuls des B soient présents à ce contact. La figure 17 présente les résultats de simulation avec un échafaudage non destructible pour des bases de temps de 200 et de 1000 unités de temps calcul.
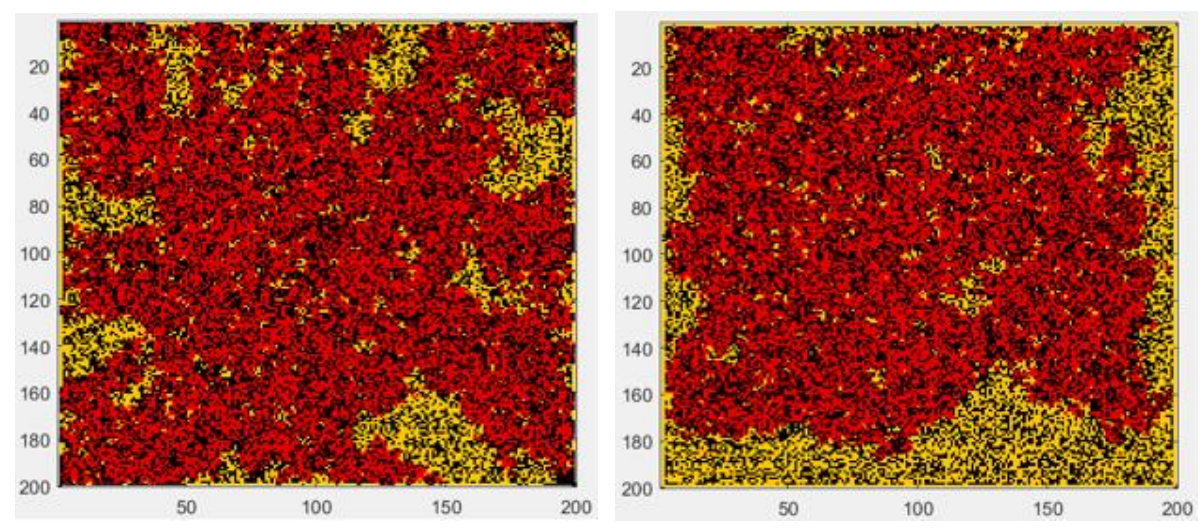

Figure 17. Résultats de simulation avec un échafaudage non destructible pour des bases de temps de 200 (à gauche) et de 1000 unités de temps calcul (à droite)

Remarque: Sur la figure 17, il est possible de mettre en évidence un biais lié à la programmation : les cellules situées en bas et pour une moindre part à droite sont favorisées dans leur développement à cause d'une question d'incrémentation. Les conséquences qualitatives ne sont cependant pas en cause.

Les B se concentrent au voisinage des bords, mais cet effet n'est visible que très proche du support et le reste de la simulation demeure parfaitement aléatoire. Cependant, cette évolution est fonction des probabilités de survie et de transformation des A et des B.

\subsubsection{Echafaudage biodégradable}

Les supports vont disparaitre au bout d'un certain temps, et de façon uniforme. Le moment auquel les échafaudages se détruisent sont fixés à l'avance. Sur une simulation de taille 200x200, les (C) 2020 ISTE OpenScience - Published by ISTE Ltd. London, UK - openscience.fr 
échafaudages seront sur le pourtour 160x160 et 180x180, et se détruisent respectivement après 250 et 400 générations. La figure 18 présente une suite d'image en temps de calculs croissants illustrant la croissance et la différenciation cellulaire.
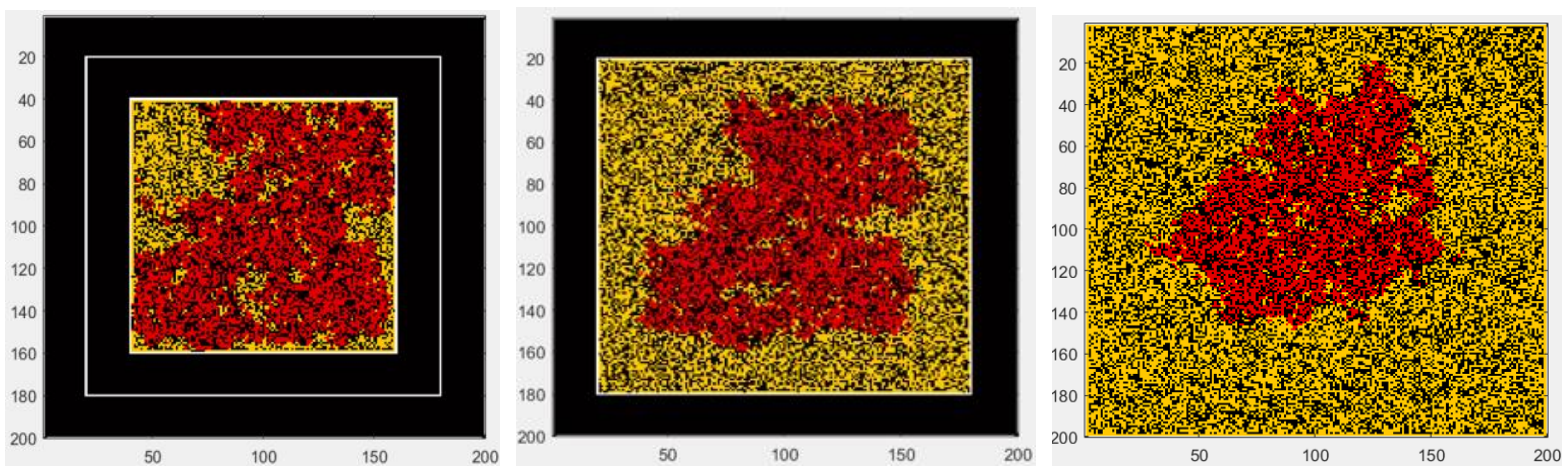

Figure 18. Influence de l'échafaudage (A détruits au contact avec le support) en fonction de sa disparition

A chaque disparition d'une couche de l'échafaudage, on observe, comme le montre la figure 19 une remise en équilibre dynamique du système.

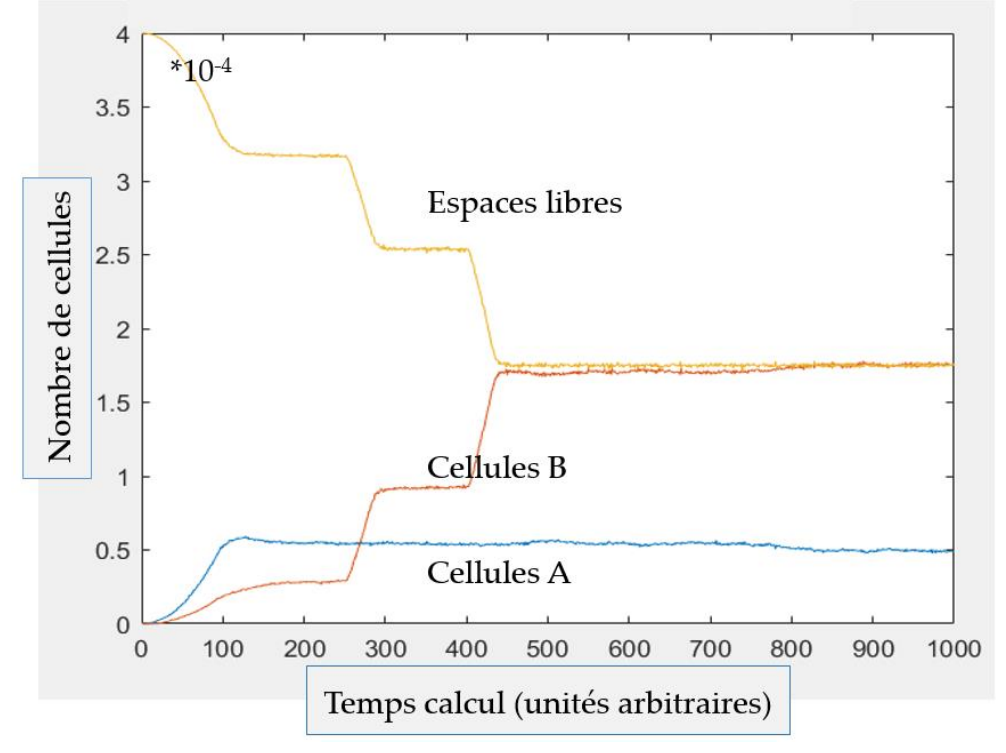

Figure 19. Evolution des populations en fonction de la dégradation des supports

Avec 2 échafaudages destructibles (en une seule opération par couche), aux temps calcul faibles, les $\mathrm{B}$ viennent recouvrir l'échafaudage. A sa destruction, les $\mathrm{B}$ continuent à se développer et le nombre des A reste relativement constant tout au long de la simulation. L'échafaudage a une réelle influence sur l'évolution du système. Ce que l'on peut observer, c'est que la position initiale de la première cellule, n'a pas d'effet remarquable sur le résultat final.

Remarque 1 : Magnan et al (2020) travaillent sur des «textiles humains » à partir de collagène qui pourraient aider à la création d'une vascularisation. Ce type d'action pourrait être envisagé car permettant de renforcer le rôle des échafaudages en leur apportant un complément de fonctionnalité. De surcroît, d'un point de vue expérimental, l'échafaudage pourrait imposer une spatialisation des micro-vaisseaux relié à un déterminisme recherché en bio-printing...

Remarque 2 : Liew Wen Loong (2020) propose de son côté des méthodes plus radicales pour préparer à la formation de micro-vaisseaux par détérioration locale du matériel biologique induit par des lasers ultra-courts permettant des transformations en profondeur dans le tissu. C'est une autre forme d'approche du déterminisme recherché. 


\subsubsection{Echafaudage biodégradable et $\alpha=5$}

En revenant aux conditions de la figure 17, la figure 22 met en lumière le fait que les $B$ forment une sorte de carapace tout autour des A. Ceux-ci n'arrivent pas à traverser cet espace. A l'issue de cette simulation on obtient un état pseudo-stable : le nombre de cellule n'évolue pratiquement pas ; la forme reste sensiblement la même. De plus, la forme et la taille de cet amas stable dépendent uniquement de la forme de l'échafaudage initial, il semble alors envisageable de modeler un amas cellulaire si l'on est capable de maîtriser les interactions intercellulaires et avec l'échafaudage. En remarque, si $\alpha$ est inférieur à 4, les A arrivent à traverser leur environnement et la simulation conduit à des résultats proches de ceux faisant l'objet de la figure 20.

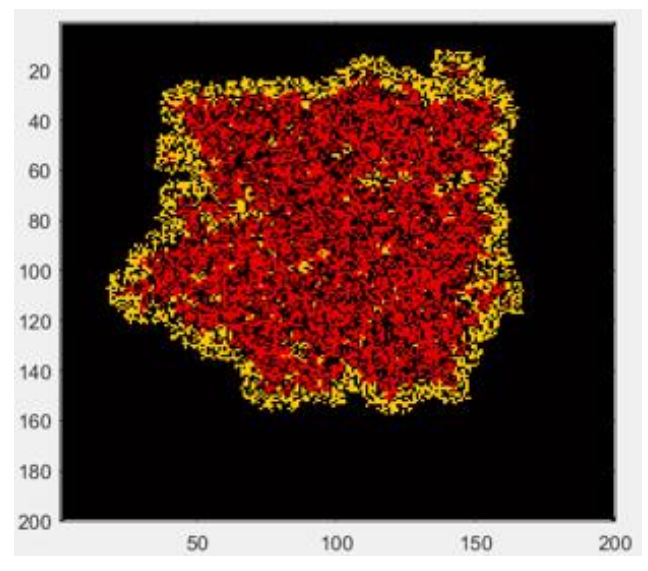

Figure 20. Cas où $\alpha=5$

\section{Discussion et conclusion}

Ce que tentent de montrer ces simulations, c'est qu'avec un nombre très restreint de paramètres, il est possible d'observer des bio-construits ayant des fonctionnalités qui peuvent mimer des comportements cellulaires. S'il en est bien ainsi dans des conditions réelles, il serait envisageable de tenter d'orienter les comportements cellulaires vers des formes susceptibles de construire des éléments de ce que pourrait être un vrai tissu biologique.

La figure 21 est là pour nous rappeler la liste des questions qu'il faudrait maîtriser avant d'arriver à un organe artificiel réalisé par bio-printing (André, 2017), à moins que la Nature fasse d'ellemême les opérations nécessaires à l'atteinte de cet objectif.

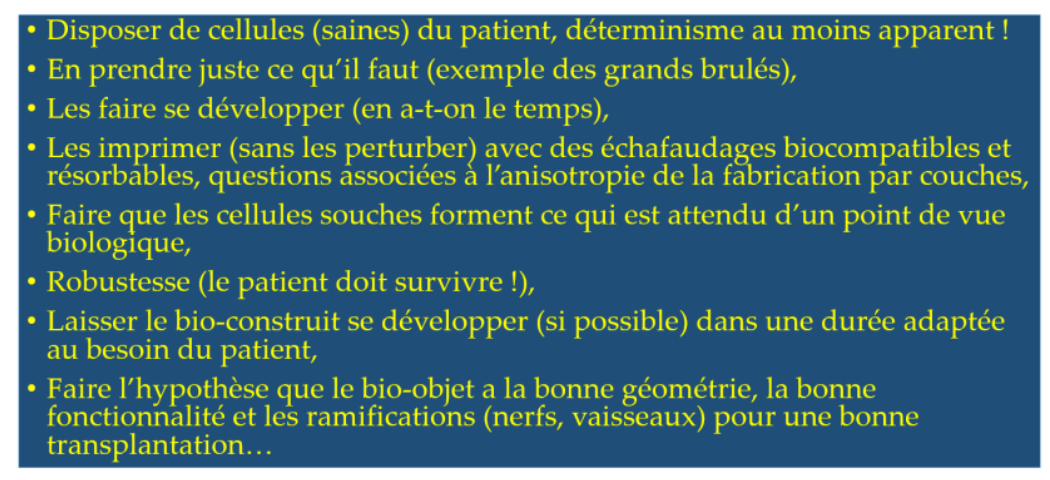

Figure 21. Rappel concernant les contraintes à satisfaire (un jour) pour le développement applicatif du bioprinting

Ce document ne traite que d'une toute petite partie d'un immense questionnement qui, de surcroît, pour des raisons de temps calcul ne traite que de systèmes en évolution à deux dimensions. En effet, si l'on peut faire évoluer (numériquement) un milieu en croissance vers l'apparition de 
zones libres qui pourraient servir à de futurs micro-vaisseaux transportant les nutriments et éliminant les déchets, rien n'est démontré sur cette même possibilité dans une simulation à 3 dimensions. Mais, il semble que cet écrit d'Atlan (2011) reste d'actualité : «Un bon modèle consistait donc à trouver une structure de connexions telle que le calcul de l'évolution du réseau à partir de cette structure le montrait se stabiliser dans les états corrects effectivement observés dans la réalité. Et c'est alors que je me suis aperçu qu'il était possible de fabriquer plusieurs modèles différents bien qu'aussi bons les uns que les autres sans que les observations disponibles puissent trancher en faveur de l'un d'entre eux ».

La figure 22 rappelle les modes de fonctionnement nécessaires dans une approche systémique... L'interdisciplinarité qui est indispensable dans ce type d'opération (après définition du cadrage du projet) est un processus dans lequel on développe une capacité d'analyse et de synthèse à partir des perspectives de plusieurs disciplines. Son objectif est de traiter une problématique dans son ensemble (principe d'intégration en ingénierie organisationnelle), en identifiant et en intégrant les relations entre les différents éléments impliqués. Il tente de synthétiser et de relier les savoirs disciplinaires et de les replacer dans un cadre systémique plus large, tout en engageant chaque membre issu d'une discipline dans le rapport à sa propre identité et sans oublier le rapport entre pratiques scientifiques et constructions identitaires.

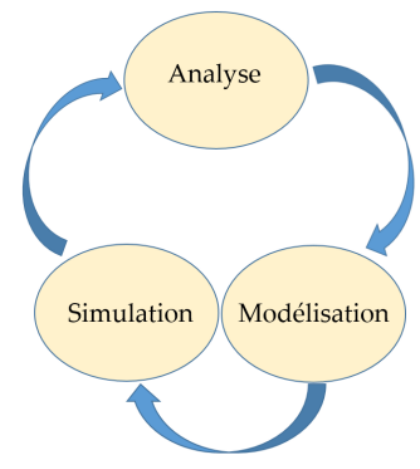

Figure 22. Le cercle « vertueux » de l'approche systémique...

C'est sans doute pour la recherche académique un moyen de tenter de « déchiffrer », à l'occasion du développement d'un thème aussi attractif que le bio-printing, des « mystères » d'un champ d'études intégrateur lui assurant, en cas de succès, une réelle légitimité, plutôt que de résoudre des problèmes «locaux » et de saisir des opportunités. Il est nécessaire d'aller un peu plus loin en ne s'appuyant plus sur des fondements inertiels classiques (parce qu'ils ont fait leurs preuves ; cf. Morange, 2012), et en proposant que l'on sorte de la spirale conservatrice suivante : plutôt que de produire des savoirs adaptés aux situations telles qu'elles se dessinent, on adapte celles-ci aux savoirs existants ou tels qu'ils sont prévus!

Ce faisant, ceux qui font peuvent naturellement mettre au point des technologies et/ou des méthodes qui répondront en partie à l'enjeu. Pourrions-nous alors trouver, comme en intelligence artificielle relativement à l'intelligence « naturelle » une bio-construction artificielle, sujet nouveau relativement à ce que sait faire la Nature... Cela pourrait ouvrir de nouveaux champs de recherche, des capacités de changer le monde, en bien comme en mal.

Si la possible révolution, dans laquelle le bio-printing nous plonge, consiste à appliquer le savoir au savoir lui-même, peut-être convient-il de laissant le mot de la fin à Jean Staune (2015) pour conclure avec cette phrase paradoxale et pessimiste : « Pourquoi étudier des problèmes qui n'ont pas de solution ou dont la solution est trop complexe pour être trouvée ? »... Est-il effectivement possible de sortir de l'épuisement des concepts pour être capables de prédire l'évolution d'un tel système complexe à partir de la connaissance de son état présent ? Y-aurait-il incomplétude et par 
conséquence indécidabilité ? Mais si l'on ne tente pas ? On en restera pour longtemps à « Imprimezmoi un organe ! Pourquoi nous n'en sommes pas encore là » ( $\mathrm{Ng}$, Chua et Shen, 2019).

\section{Bibliographie}

- AFTU (Association Pour Une Fondation Travail-Université) (2006) « Technologies convergentes, évaluations divergentes » Education Permanente, « De nouveaux enjeux pour la politique scientifique » http://www.ftu.be/documents/ep/EP-24.pdf

- André J.C. (2017) «Impression 3D : niches applicatives porteuses » Techniques de l'Ingénieur, Réf. BM7970 V1, 17pp. (2017).

- André J.C. (2017 a) "From Additive Manufacturing to 3D/4D Printing - Volume 1: From the first concept to the present applications; Volume 2: Improvement of the present technologies and constraints; Volume 3: Breakdown innovations: Programmable matter; 4D Printing and Bio-Printing” ISTE/Wiley Ed. - Londres - UK.

- Ashammakhi N., Ahadian S., Pountos I., Hu S.K., Tellisi N., Bandaru P., Ostrovidov S., Dokmeci M.R., Khademhosseini A. (2019) "In situ three-dimensional printing for reparative and regenerative therapy" Biomedical Microdevices, 21, 42 .

- Atala A., Forgacs G. (2019) “Three-Dimensional Bio-printing in Regenerative Medicine: Reality, Hype, and Future” Stems Cells Future, $\underline{8}, 744-745$.

- Atlan H. (2006) «L'organisation biologique et la théorie de l'information » Seuil Ed. - Paris.

- Atlan H. (2011) « Qu'est-ce qu'un modèle ? » Manucius Ed. - Paris.

- Ballet P., Pothet A., Misevic G., Jeannin-Girardon A., Fronville A., Rodin V. (2011) «Une approche multi-agent pour la simulation biologie cellulaire 》 https://www.google.fr/url?sa=t\&rct=j\&q=\&esrc=s\&source=web\&cd=3\&ved=0ahUKEwi70uTG-

5HOAhUHvBoKHXM9DQQQFggtMAI\&url=http\%3A\%2F\%2Fnetbiodyn.tuxfamily.org\%2FNetBioDyn\%2FTelecha rgements files\%2FNetBioDyn Exemples.pdf\&usg=AFQjCNEdtZ0gcL9r15y5srKjB-XSEYxQLQ

- Baltazar T., Merola J., Motter Catarino C., Bingchan Xie C., Kirkiles-Smith N., Lee V., Kolbeck Hotta S.Y., Dai G., Xu X., Castelo Ferreira F., Saltzman W.M., Pober J.S., Karande P. (2019) "3D bio-printing of a vascularized and perfusable skin graft using human keratinocytes, Fibroblasts, Pericytes, and Endothelial Cells" Tissue Engineering Part A, https://doi.org/10.1089/ten.tea.2019.0201

- Bar-Yam Y. (2003) « Dynamics of complex systems » Westview Press Ed. - Boulder - USA.

- Ben Amor S., Lavallée I., Bui M. (2006) « Complexité, percolation et optimisation stochastique » ROADEF”06 http://www.eurocontrol.int/eec/gallery/content/public/documents/EEC INO documents/INO documents/Benamo rLavalleeBui.pdf

- Bittner S.M., Smith B.T., Diaz-Gomez L., Hudgins C.D., Melchiorri A.J., Scott D.W., Fisher J.P., Mikos A.G. (2019) "Fabrication and mechanical characterization of 3D printed vertical uniform and gradient scaffolds for bone and osteochondral tissue engineering" Acta Biomaterialia - sous presse https://www.sciencedirect.com/science/article/pii/S1742706119302168.

- Boudon F. Chopard J., Ali O., Gilles B., Hamant O., Boudaoud A., Traas J., Godin C. (2015) “A Computational Framework for 3D Mechanical Modeling of Plant Morphogenesis with Cellular Resolution" PLOS Computational Biology, http://dx.doi.org/10.1371/journal.pcbi.1003950

- Bruno R.D., Reid J., Sachs P.C. (2019) “The revolution will be open-source: how 3D bio-printing can change 3D cell culture" Oncotarget, 10, 4724-4726.

- Chen T., Callan-Jones A., Fedorov E., Ravasio A., Brugués A., Ting Ong H., Toyama Y., Chuan Low B., Trepat X., Shemesh T., Voituriez R., Ladoux B. (2019) "Large-scale curvature sensing by directional actin flow drives cellular migration mode switching" Nature Physics, 15, 393-402.

- Chneiweiss H. (2012) «L’Homme réparé ; espoirs, limites et enjeux de la médecine régénérative » Plon Ed. - Paris.

- Collectif SMI (1995) «L'adéquation fonctionnelle comme limite des systèmes organisateurs » 58-67 in G. Théraulaz Ed. «Evolution et organisation ; hasard et contraintes dans la genèse des formes collectives » Journées de Rochebrune et ENST Ed. - Paris. http://perso.telecom-paristech.fr/ evolang/Rochebrune/Rochebrune\%201995\%20\%20Evolution\%20et\%20Organisation.pdf 
- Di Marzo Serugendo G., Gleizes M.P., Karageorgos A. (2006) "Self-organization and emergence in mas; an overview" Informatica, 30, 45-54.

- Esmaeili S., Shahali M., Kordjamshidi A., Torkpoor Z., Namdari F., Samandari S.S., Nejad M.G., Khandan A. (2019) "An artificial blood vessel fabricated by 3D printing for pharmaceutical application" Nanomedicine J., $\underline{6}$, 183-194.

- Fox-Keller E. (2009) “Organisms, Machines, and Thunderstorms, a history of Self-Organization” Historical Studies in the Natural Sciences, 38-39.

- Frank A., Gouyon P.H. (1997) “Information et complexité : questions sans réponses » La Recherche, 296, 106.

- Fried P., Iber D. (2015) "Read-out of dynamic morphogen gradients on growing domains" PloS-One 10, e0143226.

- Fromm J. (2004) “The emergence of complexity” Kassel Univ. Press Ed. - Kassel - Allemagne.

- Furfaro F. (2018) «Contrôle temporel de morphogènes pour la différentiation cellulaire » http://www.msc.univparis-diderot.fr/spip.php?article878

- Gallopin G.C., Funtowicz S., O’Connor M., Ravetz J. (2001) « La science pour le XXIème siècle : du contrat social aux fondements scientifiques. » Revue internationale des sciences sociales, 168, 239-250.

- Godesky M.D., Shreibera D.I. (2019) "Hyaluronic acid-based hydrogels with independently tunable mechanical and bioactive signaling features" Bio-interphases, 14, 061005.

- Grebenyuk S., Ranga A. (2019) "Engineering Organoid Vascularization" Frontiers in Bioengineering and Biotechnology, $\underline{7}, 39$.

- Grigoryan B., Paulsen S.J., Corbett D.C., Sazer D.W., Fortin C.L., Zaita A.J., Greenfield P.T., Calafat N.J., Gounley J.P., Ta A.H., Johansson F., Randles A., Rosenkrantz J.E., Louis-Rosenberg J.D., Galie P.A., Stevens K.R., Miller J.S. (2019) « Multivascular networks and functional intravascular topologies within biocompatible hydrogels » Science, 364, 458-464

- Guédon E., Malaquin L., André J.C. (2017) « Bio-printing - État des lieux et perspectives » Techniques de l'Ingénieur, ref. RE268 V1, 23pp. (2017).

- Guidolin D., Rebuffat P., Albertin G. (2011) “Cell-Oriented Modeling of Angiogenesis" The Scientific World Journal, $\underline{11}$, 1735-1748.

- Hanumantharao S.N., Que C., Rao S. (2019) "Self-assembly of 3D nanostructures in electro-spun poly-caprolactonepolyaniline fibers and their application as scaffolds for tissue engineering" Materialia, $\underline{6}, 100296$.

- Jacob F. (1970) « La logique du vivant » Gallimard Ed. - Paris.

- Jessus C. (2017) «Etonnant vivant - Découvertes et promesses du XXIe siècle » CNRS Ed. - Paris.

- Jiang T., Munguia-Lopez J .G. Flores-Torres S., Kort-Mascort J., Kinsella J.M. (2019) "Extrusion bio-printing of soft materials: An emerging technique for biological model fabrication featured" Applied Physics Reviews, $\underline{6}, 011310$.

- Kaern M., Elston T.C., Blake W.J., Collins J.J. (2005) "Stochasticity in gene expression: from theory to phenotypes" Nature Review of Genetics, $\underline{6}, 451-464$.

- Kahn A., Papillon F. (2005) « Le secret de la salamandre ; la médecine en quête d'immortalité » NIL Ed. - Paris.

- Karabay U., Husemoglu R.B., Eğrilmez M.Y., Havitcioglu H. (2020) "3D Printed Polylactic Acid Scaffold for Dermal Tissue Engineering Application: The Fibroblast Proliferation in vitro" Journal of Medical Innovation and Technology, 1, 51-56.

- Kim J.H., Kim I., Seol Y.J., Ko I.K., Yoo J.J., Atala A., Lee S.J. (2020) "Neural cell integration into 3D bioprinted skeletal muscle constructs accelerates restoration of muscle function" Nature Communications, 11, 1025 https://www.nature.com/articles/s41467-020-14930-9\#data-availability

- Knorr-Cetina K. (1999) "Epistemic Cultures: How the Sciences make Knowledge” Harvard University Press Ed. Cambridge - USA.

- Kupiec J.J. (2008) «L'origine des individus » Fayard Ed. - Paris.

- Kupiec J.J. (2009) « Une approche darwinienne de l'ontogenèse » https://www.cairn.info/les-mondes-darwiniensvolume-1--9782919694396-page-599.htm

- Kupiec J.J. Ed. (2013) «La vie, et alors ? - Débats passionnés d'hier et d'aujourd'hui » Belin Ed. - Paris.

- Kupiec J.J., Sonigo P. (2003) « Ni Dieu, ni gène ; pour une autre théorie de l'hérédité » Points Sciences Ed. - Paris. 
- Landau S., Moriel A., Livne A., Zheng M.H., Bouchbinder E., Levenberg S. (2018) "Tissue-Level Mechanosensitivity: Predicting and Controlling the Orientation of 3D Vascular Networks" Nano Lett., 18, 7698-7708.

- Laurent L. (2005) « Nanotechnologies et technologies convergentes : quel avenir à vingt ans ?» Le Banquet http://www.revue-lebanquet.com/nanotechnologies-et-technologies-convergentes-quel-avenir-a-vingt-ans/

- Lei M., Wang X. (2016) “Biodegradable Polymers and Stem Cells for Bio-printing” Molecules, 21, 539.

- Li T., Zhai D., Ma B., Xue J., Zhao P., Chang J., Gelinsky M., Wu C. (2019) "3D Printing of Hot Dog-Like Biomaterials with Hierarchical Architecture and Distinct Bioactivity” Advanced Science, $\underline{6}, 1901146$.

- Liew Wen Loong A. (2020) "Laser-based 3d Printing Using Bessel Beams for Tissue Engineering Applications" $\mathrm{PhD}$ of the School of Mechanical and Aerospace Engineering - Nanyang Technological University - Singapore https://dr.ntu.edu.sg/handle/10356/136772

- Lin N.Y.C., Homan K.A., Robinson S.S., Kolesky D.B., Duarte N., Moisan A., Lewis J.A. (2019) "Renal reabsorption in 3D vascularized proximal tubule models" Proceedings of the National Academy of Sciences, $\underline{116}$, 5399-5404.

- Lipson H., Kurman M. (2014) «Impression 3D : la prochaine révolution » Wiley \& First Interactive Ed. - Paris.

- Listek V. (2019) “The Year in Review: Bio-printing in 2019” https://3dprint.com/261238/the-year-in-review-bioprinting-in-2019/

- Magnan L., Labrunie G., Fénelon M., Dusserre N., Foulc M.P., Lafourcade M., Svahn I., Gontier E., Vélez J.H., McAllister T.N., L'Heureux N. (2020) "Human textiles: A cell-synthesized yarn as a truly "bio" material for tissue engineering applications" Acta Biomaterialia, 105, 111-120.

- Mathieu N., Schmid A.F. (2014) «Reconsidérer le lien entre modélisation et interdisciplinarité » 7-18 in N. Mathieu et A.F. Schmid Ed. « Modélisation et interdisciplinarité ; six disciplines en quête d'épistémologie » Quae Ed. Paris.

- McCune M., Shafiee A., Forgacs G., Kosztin I. (2014) "Predictive modeling of post bio-printing structure formation" Soft Matter, 10, 1790-1800.

- Melchels F.P.W., Domingos M.A.N., Klein T.J., Maldo J., Bartolo P.J., Hutmacher D.W. (2012) “Additive manufacturing of tissues and organ"” Progress in Polymer Science, 37, 1079-1084.

- Merks R.M.H., Koolwijk P. (2009) "Modeling Morphogenesis in silico and in vitro: Towards Quantitative, Predictive, Cell-based Modeling” Math. Model. Nat. Phenom., 4, 149-171. DOI: 10.1051/mmnp/20094406.

- MIT (2016) "Convergence: The Future of Health (A novel research strategy that integrates biomedical knowledge with advanced engineering skills and expertise from physical, computational, and mathematical sciences)"

http://static1.squarespace.com/static/570561df4d088ef84160338a/t/576bf712ff7c50b7fcb48482/1466693398318/Converge nce-The-Future-of-Health-2016-Report.pdf

- Morange M. (2012) «Les secrets du vivant ; contre la pensée unique en biologie » La découverte Ed. - Paris.

- Neagu A., Kosztin I., Jakab K., Barz B., Neagu M., Jamison R. (2006) "Computational modeling of tissue selfassembly" Modern Physics Letters B, 20, $1217\{1231$; http://organprint.missouri.edu/www/fibr-pub/neagu061217.pdf

- Ng W.L., Chua C.K., Shen Y.F. (2019) "Print me an organ! Why we are not there yet" Progress in Polymer Science, 97, 101145.

- Nuñez Bernal P., Delrot P., Loterie D., Li Y., Malda J., Moser C., Levato R. (2019) "Volumetric Bio-printing of Complex Living-Tissue Constructs within Seconds" Advanced Materials, 31, 1904209.

- Okuda S·, Inoue Y., Eiraku M., Adachi T., Sasai Y. (2016) "Modeling cell apoptosis for simulating threedimensional multicellular morphogenesis based on a reversible network reconnection framework » Bio-mechanic Modeling \& Mechano-biology, 15, 805-816.

- Osaghae E. (2019) “Bio-printing 101 Part 18 - Pharmaceutical Testing” https://3dprint.com/243760/bio-printing-101part-18-pharmaceutical-testing/ et autres parties.

- Ottino J.M. (2004) “Complex systems” American Institute of Chemical Engineering Journal, 49, 292-299.

- Ozbolat I., Hospodiuk M. (2016) "Current advances and future perspectives in extrusion based bio-printing" Biomaterials, $\underline{76}, 321-343$. 
- Ozbolat I.T., Peng W., Ozbolat V. (2016) “Application areas of 3D bio-printing” Drug Discovery Today, 21, 12571271.

- Pareto V. (1965) «Les systèmes socialistes » Droz Ed. - Genève - Suisse.

- Park J.S., Suryaprakash S., Lao Y.H., Leong K.W., (2015) "Engineering mesenchymal stem cells for regenerative

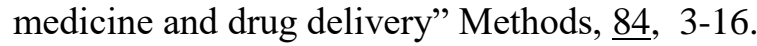

- Parrochia D. (1994) «Le statut épistémologique de la vie artificielle»164-185 in F. Tinland «Ordre biologique Ordre technologique » Champ Vallon Ed. - Paris.

- Pasturel A., Strale P.O., Studer V. (2019) "Tailoring 3D cell culture templates with common hydrogels" BioRxiv preprint Doi: https://doi.org/10.1101/370882.

- Pavé A. (2012) « Modélisation des systèmes vivants : De la cellule à l'écosystème » Lavoisier Ed. - Paris.

- Peels J. (2019) "Bio-printing“ https://3dprint.com/251674/charles-river-associates-international-on-bio-printing/

- Peng W., Unutmaz D., Ozbolat I.T. (2016) "Bio-printing towards Physiologically Relevant Tissue Models for Pharmaceutics" Trends in Biotechnology, 34, 722-732.

- Peters M.D., Wittwer L.D., Stopka A., Barac D., Lang C., Iber D. (2018) "Simulation of morphogen and tissue dynamics" arXiv:1806.041

- Richards D., Jia J., Yost M., Markwald R., Mei Y. (2017) “3D Bio-printing for Vascularized Tissue Fabrication” Annals of Biomedical Engineering, 45, 132-147.

- Rothstein J. (1962) «Information and organization as the language of the operational viewpoint » The Philosophy of Science, $\underline{29}, 406-411$.

- Samsara (2016) "Primary human hepatic stellate cells" http://www.samsarasciences.com/

- Seijkens D., Olinga P., Euverink G.J.W., Melgert B.N. (2018) "Putting INVITROM in the market - An advice on the opportunities for INVITROM for funding, membership and content" http://fse.studenttheses.ub.rug.nl/18100/1/mBMS 2018 SeijkensDA.pdf

- Schmid A.F. (2010) « Epistémologie et éthique technologique » https://hal-univ-lyon3.archivesouvertes.fr/file/index/docid/707832/filename/epistemo ethique schmid.pdf

- Senior J.J., Cooke M.E., Grover L.M., Smith A.M. (2019) "Fabrication of Complex Hydrogel Structures Using Suspended Layer Additive Manufacturing (SLAM)" Fabrication of Complex Hydrogel Structures Using Suspended Layer Additive Manufacturing (SLAM)" Advances in Functional Materials, 1904845.

- Seth D., Cheldize K., Brown D., Freeman E.F. (2017) "Global Burden of Skin Disease: Inequities and Innovations" Current Dermatology Report, $\underline{6}, 204-210$.

- Skardal A., Atala A. (2015) "Biomaterials for integration with 3D bio-printing" Annals of Biomedical Engineering, $\underline{43}, 730-746$.

- Skylar-Scott M.A., Uzel1 S.G.M., Nam L.L., Ahrens J.H., Truby R.L., Damaraju S., Lewis J.A. (2019) "Biomanufacturing of organ-specific tissues with high cellular density and embedded vascular channels" Science Advances, $\underline{5}$, eaaw2459.

- Sommer P. (2016) «Les tissus humains, entre biologie et mécanique - propos d'un biologiste » MECAMAT CNRS (18-22 janvier) - Aussois.

- Staune J. (2015) «Les clés du futur ; réinventer ensemble la société, l'économie et la science» Plon Ed. - Paris.

- Sun H., Hu C., Zhou C., Wu L., Sun J., Zhou X., Xing F., Long C., Kong Q., Liang J., Fan Y., Zhang X. (2020) “3D printing of calcium phosphate scaffolds with controlled release of antibacterial functions for jaw bone repair" Materials \& Design, $\underline{189}, 108540$.

- Sun M., Zaman M.H. (2017) "Advanced Review Modeling, signaling and cytoskeleton dynamics: integrated modeling-experimental frameworks in cell migration" WIREs Syst. Biol. Med., 9, e1365. doi: 10.1002/wsbm. 1365 .

- Tamay D.G., Usal1 T.D., Alagoz A.S., Yucel D., Hasirci N., Hasirc V. (2019) “3D and 4D Printing of Polymers for Tissue Engineering Applications" Frontiers in Bioengineering and Biotechnology, 7, 164.

- Thuillier F. (2018) «Bio-printing : vers une modélisation mathématique » PRD ENSIC- Nancy. 
- Von Foerster H. (1960) «Sur les systèmes auto-organisateurs et leurs environnements » 31-50 in M.C. Yovits et S. Cameron Ed. "Self-Organizing Systems" Pergamon Press Ed. - Londres - UK.

- Wang Y., Sun Q. (2013) "Modeling and simulations of multicellular aggregate self-assembly in bio-fabrication using kinetic Monte Carlo methods" Soft Matter, $\underline{9}, 2172$.

- Wimmer R.A., Leopoldi A., Aichinger M3. Wick N., Hantusch B., Novatchkova M., Taubenschmid J., Hämmerle M., Esk C., Bagley J.A., Lindenhofer D., Chen G., Boehm M., Agu C.A., Yang F., Fu B., Zuber J., Knoblich J.A., Kerjaschki D., Penninger J.M. (2019) "Human blood vessel organoids as a model of diabetic vasculopathy" Nature, $\underline{565}$, 505-510.

- Wolpert L., Tickle C., Arias A.M. (2017) «Biologie du développement - Les grands principes » Dunod Ed. - Paris.

- Zheng Y., Han M.K.L., Jiang Q., Li B., Fengab J., del Campo A. (2020) “““ 4D hydrogel for dynamic cell culture with orthogonal, wavelength-dependent mechanical and biochemical cues" Material Horizons, $\underline{7}, 111-116$.

- Zhong Q. (2019) «Bio-printing 2 : vers une modélisation mathématique » Projet de Recherche et Développement de l'ENSIC - Nancy.

\section{Annexe : Choix du langage et programmes utilisés ; organigramme}

\section{Langage utilisé}

1- L'utilisation de matrices sur Matlab est simple à mettre en place. Le système étant une grille pouvant être une cellule ou du vide, cela est facilement représentable par une matrice avec des valeurs de 0 pour une case vide, 1 pour une cellule A, 2 pour une cellule B et 3 pour un échafaudage. La manipulation des matrices et leur transformation est relativement évidente et facile à coder. De plus, la création de matrice de probabilité est facilement définissable comparé à d'autre logiciels de programmation.

2- La représentation graphique est très simple grâce à la fonction imagesc(). Cette fonction sert initialement à créer une image à partir d'une matrice, dont les couleurs sont fonctions des valeurs dans la matrice. Les couleurs sont échelonnées (un peu comme une caméra thermique dont les valeurs de température les plus élevées sont autour du blanc et du rouge, et les températures les plus froides autour du bleu et du noir). C'est pour cette raison que les couleurs entre les figures sont différentes, plus il y a de valeurs différentes dans la matrice (rappel 0-vide ; 1-A ; 2-B ; 3-mur), plus les couleurs vont changer et s'échelonner. La couleur n'a donc finalement aucune importance sur l'évolution, tout dépend du nombre de valeurs différentes dans la matrice.

3)- Grâce à la fonction pause(), il est possible de stopper le code pour un bref instant $(0.01$ seconde) afin d'observer la progression des cellules (nombre, différenciation) entre chaque itération. Cela permet une observation très visuelle du comportement des cellules et de pouvoir déceler des tendances.

\section{Organigramme}

Il fait l'objet de la figure 23, avec différents éléments représentés ci-après.

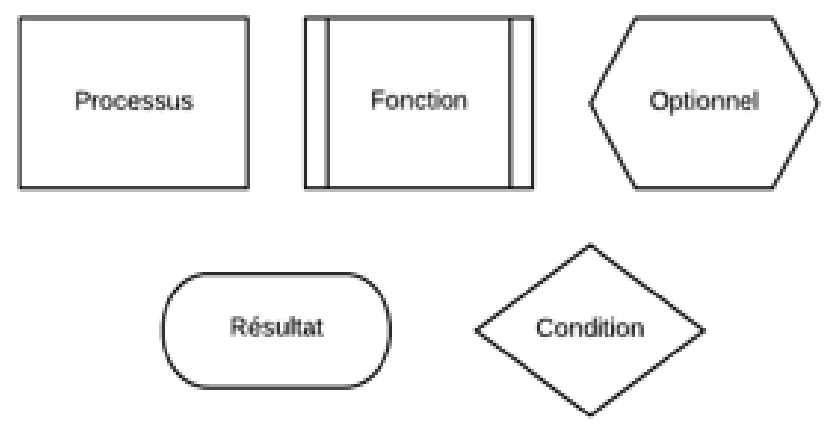




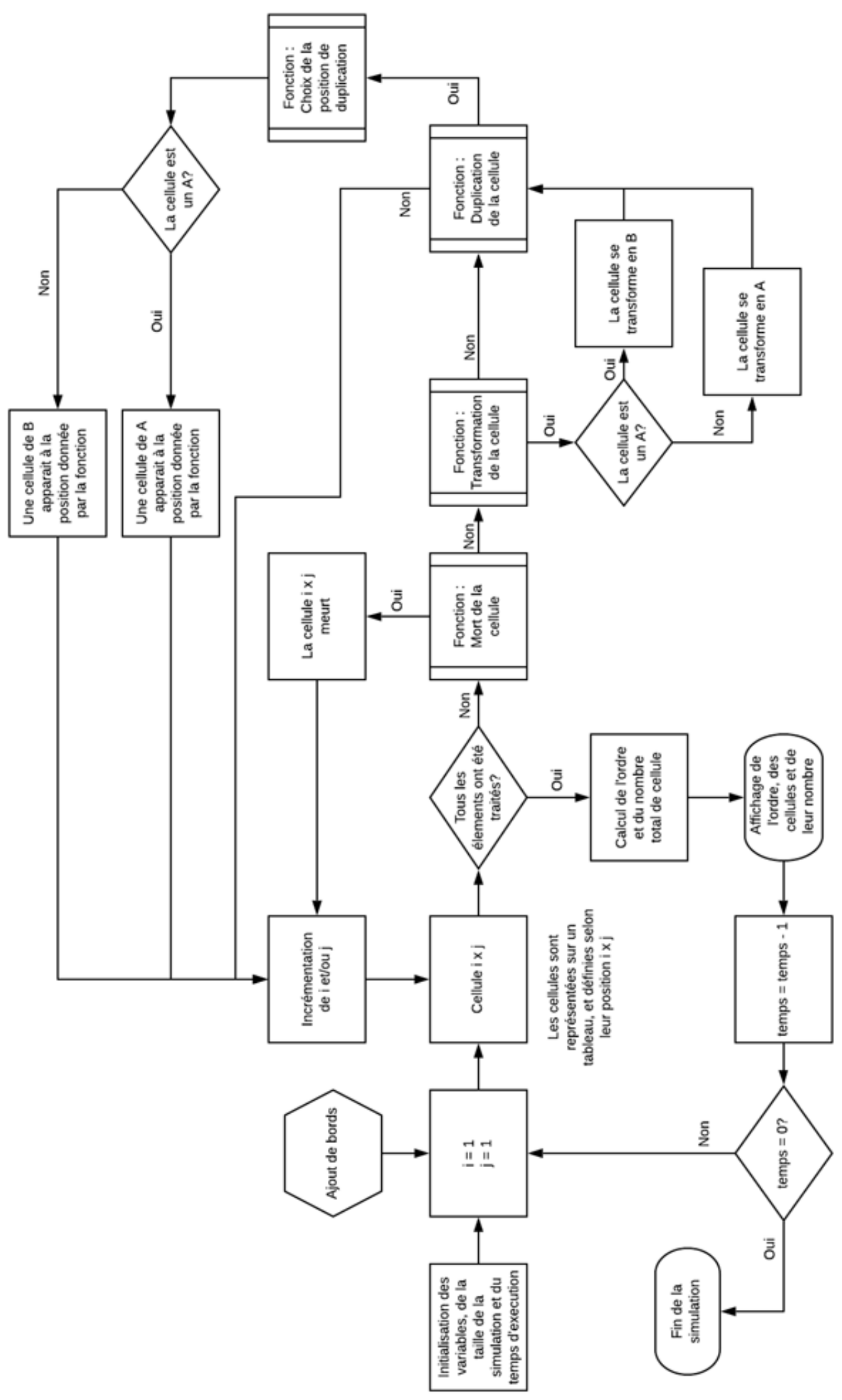

Figure 23. Organigramme de base utilisé 